\title{
Exogenous plant MIR168a specifically targets mammalian LDLRAP1: evidence of cross-kingdom regulation by microRNA
}

Lin Zhang ${ }^{1, *}$, Dongxia Hou ${ }^{1, *}$, Xi Chen ${ }^{1, *}$, Donghai $\mathrm{Li}^{1,{ }^{*}}$, Lingyun Zhu ${ }^{1,2}$, Yujing Zhang ${ }^{1}$, Jing Li ${ }^{1}$, Zhen Bian ${ }^{1}$, Xiangying Liang ${ }^{1}$, Xing Cai ${ }^{1}$, Yuan Yin $^{1}$, Cheng Wang ${ }^{1}$, Tianfu Zhang ${ }^{1}$, Dihan Zhu ${ }^{1}$, Dianmu Zhang ${ }^{1}$, Jie Xu ${ }^{1}$, Qun Chen ${ }^{1}$, Yi Ba ${ }^{3}$, Jing Liu ${ }^{1}$, Qiang Wang ${ }^{1}$, Jianqun Chen ${ }^{1}$, Jin Wang ${ }^{1}$, Meng Wang ${ }^{1}$, Qipeng Zhang ${ }^{1}$, Junfeng Zhang ${ }^{1}$, Ke Zen ${ }^{1}$, Chen-Yu Zhang ${ }^{1}$

${ }^{I}$ Jiangsu Engineering Research Center for microRNA Biology and Biotechnology, State Key Laboratory of Pharmaceutical Biotechnology, School of Life Sciences, Nanjing University, 22 Hankou Road, Nanjing, Jiangsu 210093, China; ${ }^{2}$ Department of Chemistry and Biology, School of Science, National University of Defense Technology, Changsha, Hunan 410073, China; ${ }^{3}$ Tianjin Medical University Cancer Institute and Hospital, Huanhuxi Road, Tiyuanbei, Tianjin 300060, China

Our previous studies have demonstrated that stable microRNAs (miRNAs) in mammalian serum and plasma are actively secreted from tissues and cells and can serve as a novel class of biomarkers for diseases, and act as signaling molecules in intercellular communication. Here, we report the surprising finding that exogenous plant miRNAs are present in the sera and tissues of various animals and that these exogenous plant miRNAs are primarily acquired orally, through food intake. MIR168a is abundant in rice and is one of the most highly enriched exogenous plant miRNAs in the sera of Chinese subjects. Functional studies in vitro and in vivo demonstrated that MIR168a could bind to the human/mouse low-density lipoprotein receptor adapter protein 1 (LDLRAP1) mRNA, inhibit LDLRAP1 expression in liver, and consequently decrease LDL removal from mouse plasma. These findings demonstrate that exogenous plant miRNAs in food can regulate the expression of target genes in mammals.

Keywords: microRNA; MIR168a; LDLRAP1; low-density lipoprotein; microvesicle; cross-kingdom

Cell Research (2012) 22:107-126. doi:10.1038/cr.2011.158; published online 20 September 2011

\section{Introduction}

MicroRNAs (miRNAs), a class of 19-24 nucleotide long non-coding RNAs derived from hairpin precursors, mediate the post-transcriptional silencing of an estimated $30 \%$ of protein-coding genes in mammals by pairing with complementary sites in the $3^{\prime}$ untranslated regions (UTRs) of target genes [1,2]. miRNAs have been widely shown to modulate various critical biological processes, including differentiation, apoptosis, proliferation, the im-

*These four authors contributed equally to this work.

Correspondence: Chen-Yu Zhang ${ }^{\mathrm{a}}$, Ke Zen ${ }^{\mathrm{b}}$, Junfeng Zhang ${ }^{\mathrm{c}}$

a'E-mail: cyzhang@nju.edu.cn

${ }^{b}$ E-mail: kzen@nju.edu.cn

${ }^{\circ} E-m a i l:$ jfzhang@nju.edu.cn

Received 11 August 2011; revised 23 August 2011; accepted 26 August 2011; published online 20 September 2011 mune response, and the maintenance of cell and tissue identity $[1,2]$. Dysregulation of miRNAs has been linked to cancer and other diseases [3, 4]. Recently, we and others found that mammalian miRNAs exist stably in the sera and plasma of humans and animals $[5,6]$. Specific serum miRNA expression profiles show a great potential to serve as a novel class of biomarkers for the diagnosis of cancer and other diseases [5,6]. Numerous reports have subsequently shown that unique expression patterns of circulating miRNAs reflect various physiological and pathological conditions [7-12].

We next characterized the possible carrier of circulating miRNAs. Microvesicles (MVs) are small vesicles that are shed from almost all cell types under both normal and pathological conditions [13, 14]. They bear surface receptors/ligands of the original cells and have the potential to selectively interact with specific target cells and mediate intercellular communication by transporting 
bioactive lipids, mRNA, or proteins between cells [13, 14]. Our recent results demonstrated that MVs from human plasma are a mixture of microparticles, exosomes, and other vesicular structures and that many types of MVs in human plasma contain miRNAs [15]. These findings were in agreement with the recent reports by other investigators that exosomes from cultured cells served as physiological carriers of miRNAs $[16,17]$. Our further studies demonstrated that miRNAs could be selectively packaged into MVs and actively delivered into recipient cells where the exogenous miRNAs can regulate target gene expression and recipient cell function [15]. Thus, secreted miRNAs can serve as a novel class of signaling molecules in mediating intercellular communication [15]. The novel and important functions of the secreted miRNAs were also reported by many other groups [18-21]. The identification of circulating miRNAs, mainly delivered by cell-secreted MVs, as stable and active signaling molecules opens a new field of research in intercellular and interorganelle signal transduction.

In the present study, we were surprised to detect exogenous plant miRNAs in the serum and plasma of human and animals. Over half of plant miRNAs detected in serum and plasma are present in MVs. Further in vitro and in vivo analysis demonstrated for the first time that food-derived exogenous plant MIR168a can pass through the mouse gastrointestinal (GI) track and enter the circulation and various organs especially the liver where it cross-kingdomly regulates mouse LDLRAP1 protein expression and physiological condition.

\section{Results}

Plant miRNAs are present in human and animal sera and organs

Upon investigation of the global miRNA expression profile in human serum, we found that exogenous plant miRNAs were consistently present in the serum of healthy Chinese men and women. As shown in Figure 1A and Supplementary information, Table S1, Solexa sequencing revealed $\sim 30$ known plant miRNAs in Chinese healthy donors, among which MIR156a and MIR168a showed considerable levels of expression (the mean Solexa reads/total mammalian miRNAs $>0.005$ ). A comparison of the Solexa reads of plant miRNAs between male and female sera revealed no significant difference for the majority of plant miRNAs. As shown in the Pearson's correlation scatter plot comparing plant miRNAs in male and female sera, the Pearson's correlation coefficient $(R)$ was close to 1 ( $R=0.9002$; Supplementary information, Figure S1A). These healthy Chinese women and men had no metabolic dysfunctions, and their main diet was rice. Plant miRNAs were also detected in the sera of other animals, such as calves, whose diet was mainly green rich fodder (Supplementary information, Table S2). The significant differences in plant miRNA profiles between human and calf sera are shown in Supplementary information, Figure S1B, with a Pearson's correlation coefficient $(R)$ of 0.3951 . Furthermore, although the number of plant miRNAs was about $5 \%$ of mammalian miRNAs in human and calf sera (Supplementary information, Figure S1C and S1E), there were fewer absolute sequencing reads from the plant miRNAs (Supplementary information, Figure S1D and S1F). To confirm the Solexa data, the levels of MIR168a and MIR156a, the two plant miRNAs with the highest levels in the sera of Chinese subjects, and MIR166a, a plant miRNA with modest level, were assessed by a stem-loop quantitative reverse transcription polymerase chain reaction (qRT-PCR) assay. MIR161, whose expression level was undetectable, served as a negative control. First, the dynamic range and sensitivity of the qRT-PCR assay for measuring plant miRNAs in serum were evaluated. A series of synthetic plant miRNA oligonucleotides with known concentrations were reverse transcribed and amplified to generate a standard curve. As shown in Supplementary information, Figure S1G, these plant miRNAs had a linear semilogarithmic plot in a range from $1 \mathrm{fM}$ to $10^{4} \mathrm{fM}$. In other words, plant miRNAs at a level as low as $1 \mathrm{fM}$ can be efficiently examined by this assay. The absolute concentration of each miRNA was then calculated according to the standard curve. As can be seen in Figure 1B, the tested plant miRNAs were clearly present in sera from humans, mice, and calves. Moreover, when compared to the endogenous mammalian miRNAs known to be stably present in animal serum $[5,15]$, these plant miRNAs were relatively lower, but in a similar concentration range. The levels of plant miRNAs in various serum samples were about one-tenth of that of endogenous miR-16 (Figure 1B, insert), one of the major endogenous miRNAs in animal serum $[5,6]$. Furthermore, the presence of plant miRNAs in human and animal serum was confirmed by semi-quantitative RT-PCR and northern blotting (Figure 1C and 1D). To determine whether the MIR156a, MIR168a, and MIR166a identified in serum are genuine plant miRNAs, we treated the total small RNA isolated from human serum with sodium periodate (oxidizing agent). Plant miRNAs are 2'-O-methyl modified on their terminal nucleotide, which renders them resistant to periodate [22]. In contrast, mammalian miRNAs with free $2^{\prime}$ and $3^{\prime}$ hydroxyls are sensitive to periodate. Since oxidized terminal nucleotides can not be ligated to the cloning adapter, a comparison of deep sequencing libraries before and after oxidation allows 
A

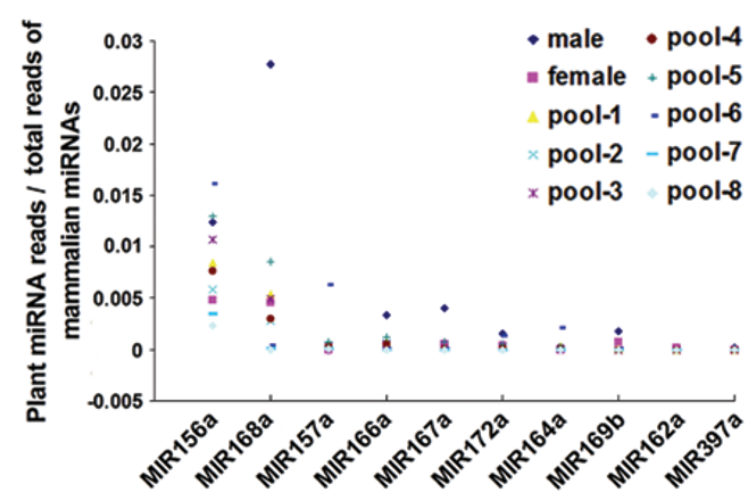

B

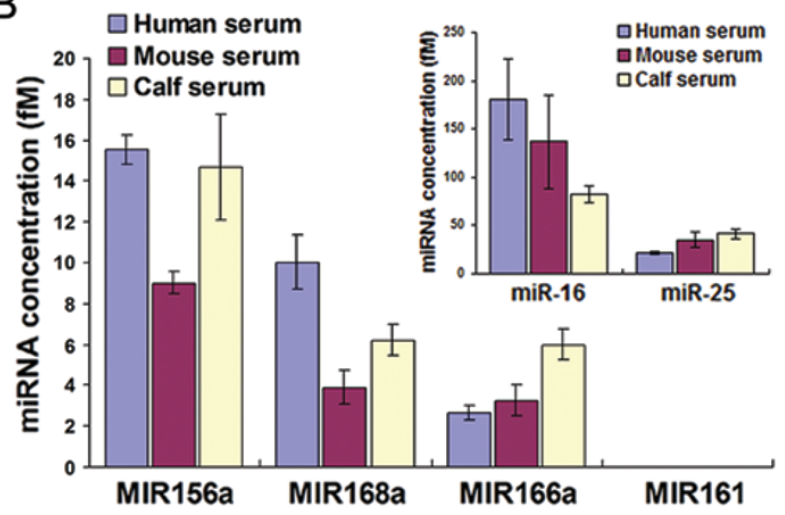

C

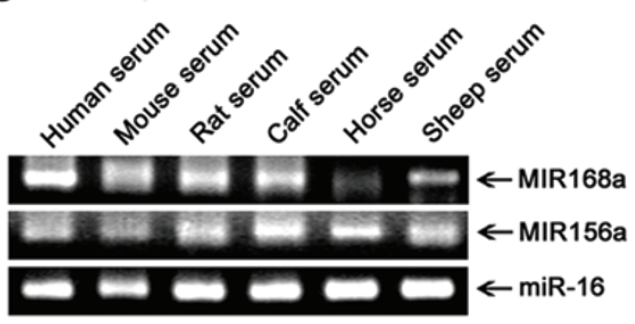

D
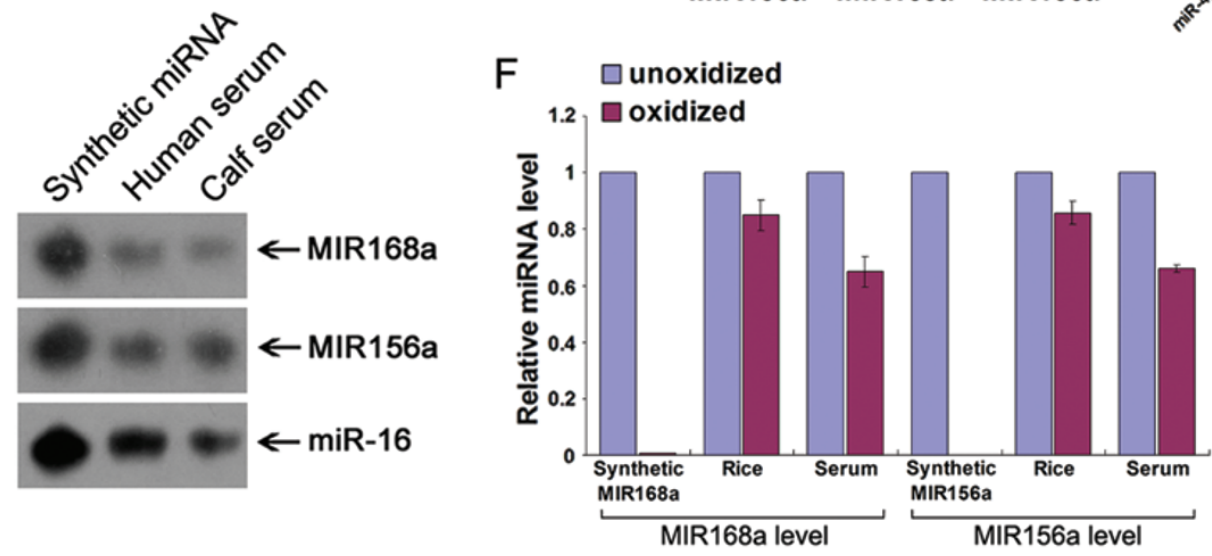

G

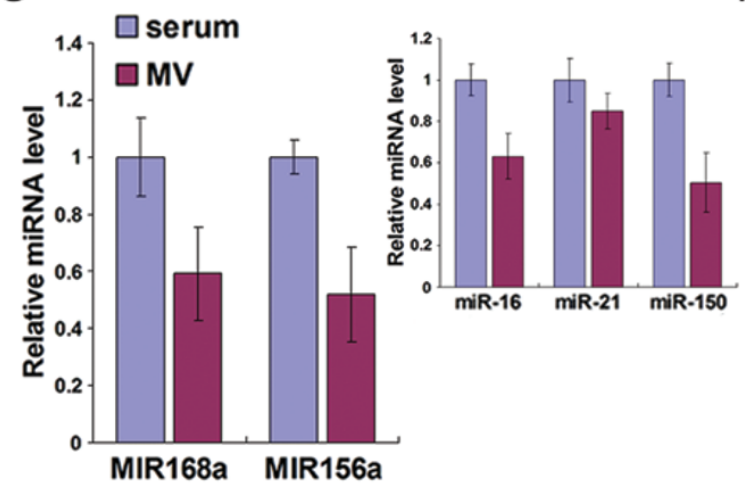

$\mathrm{H}$
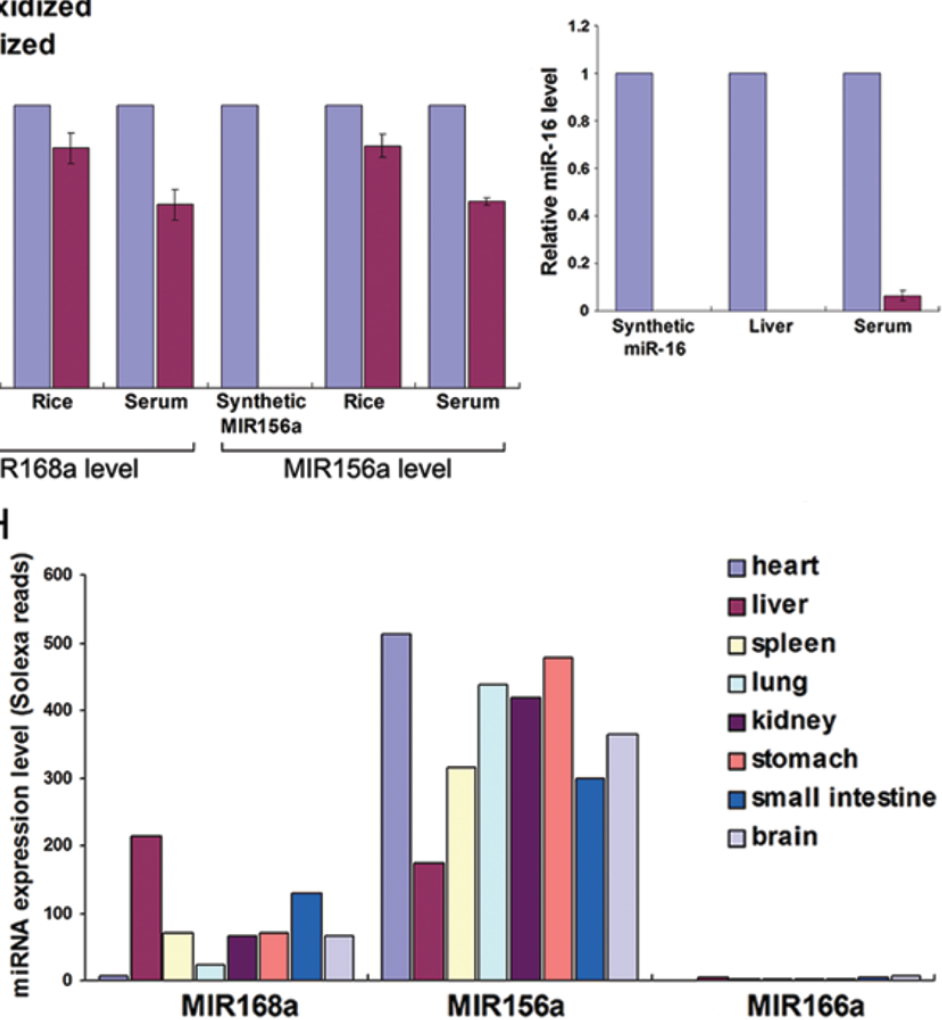

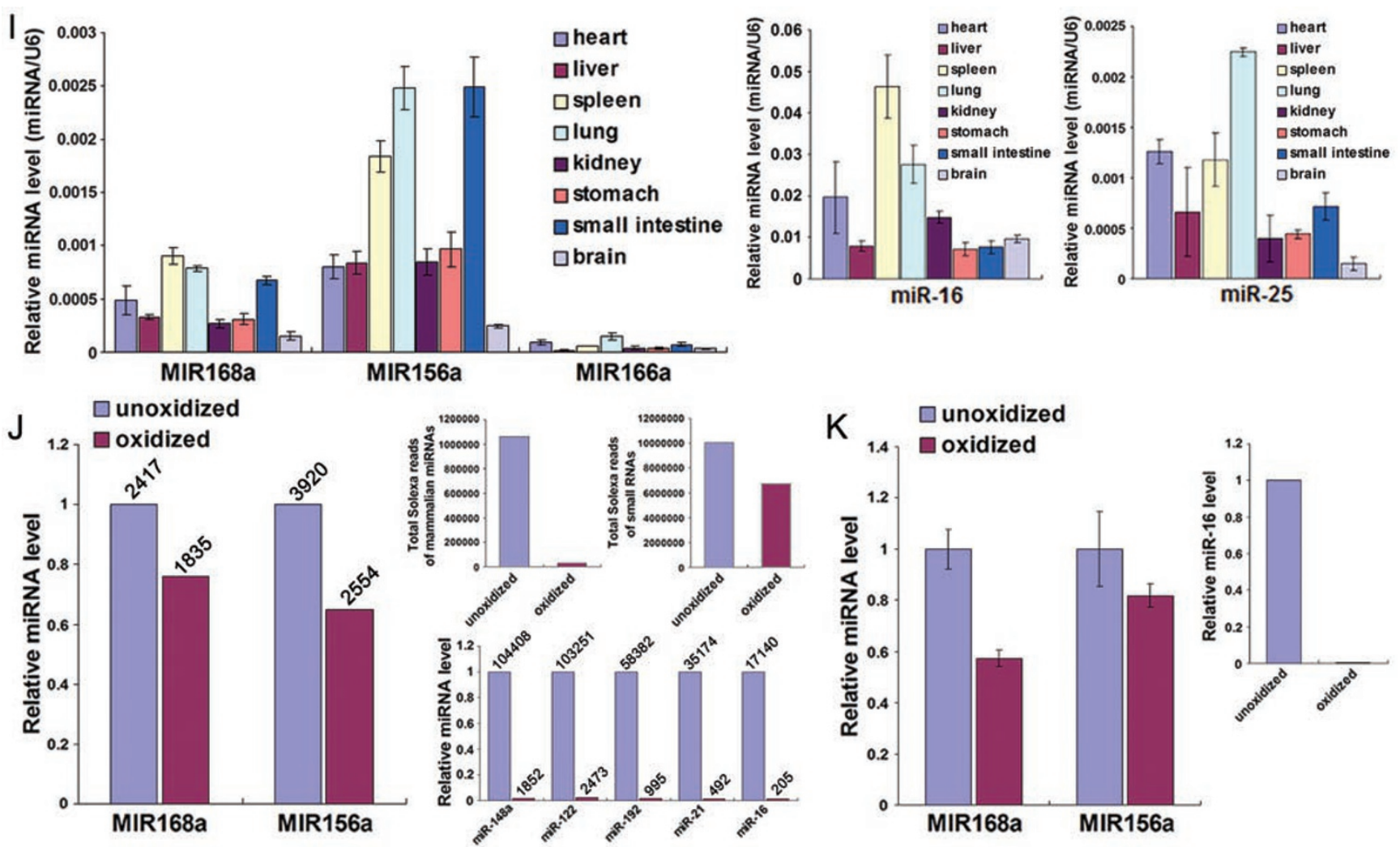

Figure 1 Plant miRNAs are present in human and animal sera and organs. (A) The levels (Solexa reads) of 10 plant miRNAs detected by Solexa sequencing in sera from healthy Chinese men and women, and eight pooled samples (each pooled from 10 healthy Chinese subjects). For normalization, the sequencing frequency of each plant miRNA was normalized to the total amount of mammalian miRNAs. (B) The absolute levels of plant miRNAs in the sera of various mammals detected by qRTPCR $(n=6)$. Endogenous animal miRNAs, miR-16, and miR-25 serve as controls (insert). (C) Semi-quantitative RT-PCR analysis of the indicated miRNAs in the serum from human, mouse, rat, calf, horse, and sheep. Accurate amplification of each miRNA was confirmed by Sanger-based method to sequence the PCR products. (D) Northern blotting analysis of the expression levels of MIR168a, MIR156a, and miR-16 in human serum (100 ml) and calf serum (40 ml). Synthetic MIR168a, MIR156a, and miR-16 (1 pmol) served as positive controls. (E) Equal amount of total small RNAs (each from $100 \mathrm{ml}$ of human serum) were treated with/without sodium periodate. After the reactions, the RNAs were purified and then subjected to Solexa sequencing. Solexa reads of the plant miRNAs in oxidized and unoxidized groups were compared. Total and individual mammalian miRNAs were compared to serve as controls (insert). The absolute Solexa reads of miRNAs are indicated. (F) Equal amount of synthetic plant miRNAs (without 2'-O-methylated 3' ends) and total small RNAs isolated from rice and human serum were treated with/without sodium periodate. After the reactions, the RNAs were subjected to qRT-PCR with the miScript PCR system. Synthetic miR-16 and total small RNAs isolated from mouse liver and human serum were treated as above. MiR-16 expression levels with/without oxidation were compared to serve as controls (insert). (G) The levels of plant miRNAs detected by qRT-PCR in MVs isolated from C57BL/6J mouse plasma $(n=4)$. $(\mathbf{H})$ The levels of plant miRNAs detected by Solexa sequencing in various organs of C57BL/6J mice. (I) The levels of plant miRNAs detected by qRT-PCR in various organs of C57BL/6J mice (normalized to U6; $n=6$ ). As before, miR-16 and miR-25 serve as controls (insert). ( $\mathrm{J}$, K) The levels of plant miRNAs detected by Solexa sequencing (J) and qRT-PCR (K) in mouse liver after oxidation. Similarly, mammalian miRNAs serve as controls (insert). The absolute Solexa reads of miRNAs are indicated.

assessing whether the small RNAs tested bear 2'-Omethylated $3^{\prime}$ ends $[23,24]$. Indeed, as shown in Figure $1 \mathrm{E}$, most mammalian miRNAs in human serum, such as miR-423-5p, miR-320a, miR-483-5p, miR-16, and miR-221, had an unmodified 2', 3' hydroxyls and were therefore oxidized and failed to be sequenced by Solexa.
In contrast, MIR156a, MIR168a, and MIR166a in human serum remained unchanged with this treatment and were sequenced by Solexa, suggesting that they bear 2'-Omethylated 3' ends and are therefore genuine plant miRNAs. Moreover, the methylation status of small RNAs can be evaluated by qRT-PCR with miScript PCR system 
after treating the small RNAs with sodium periodate. This method blocks ligation of a poly(A) tail to small RNAs bearing $2^{\prime}, 3^{\prime}$ hydroxy termini, preventing them from being reverse transcribed and amplified. Consistent with the results of oxidized deep sequencing, MIR156a and MIR168a in rice and serum showed high levels of resistance to periodate and could be amplified, whereas miR-16 in liver and serum was oxidized and unable to be detected (Figure 1F). We next determined the portion of circulating plant miRNAs in MVs compared to MV-free plasma. Similar to most endogenous miRNAs, such as miR-16, miR-21, and miR-150 (Figure 1G, insert), plant MIR168a and MIR156a were primarily detected in MVs in C57BL/6J mouse plasma (Figure 1G). Further study revealed that plant MIR168a and MIR156a were detected in various mouse tissues, including liver, small intestine, and lung (Figure 1H and 1I). Interestingly, MIR166a was nearly undetectable in various mouse tissues, although it was present in the serum. In these studies, the levels of miRNAs were normalized to U6 snRNA and other small RNAs such as snoRNA146 and snoRNA251. As shown in Supplementary information, Figure S2A, the expression levels of U6 and small RNAs were approximately equivalent between different mouse tissues. Moreover, no plant pre-miRNA was detected by semi-quantitative RT-PCR in human and animal sera and tissues (Supplementary information, Figure S2B). The presence of genuine plant miRNAs in mouse tissues was further demonstrated by oxidized deep sequencing and validated by qRT-PCR with miScript PCR system. As shown in Figure $1 \mathrm{~J}$ and $1 \mathrm{~K}$, mammalian miRNAs in mouse liver were oxidized and undetectable by both Solexa sequencing and qRT-PCR assay with miScript PCR system. In contrast, MIR168a and MIR156a in mouse liver were resistant to oxidation by periodate.

The exogenous mature plant miRNAs in food can pass through mouse GI tract and enter the sera and organs

Next, we sought to identify the source of these exogenous plant miRNAs in the sera and organs of mammals. Given that plant MIR168a and MIR156a have been reported to be enriched in various plants, including rice (Oryza sativa) and crucifers (Brassicaceae) [25, 26], and that rice is the main stable food in China, we hypothesized that plant miRNAs in the sera and tissues of humans and animals are primarily a result of food intake. To test this hypothesis, the expression levels and stability of plant miRNAs in various foods were investigated. As shown in Supplementary information, Table S3, plant MIR168a, MIR156a, and MIR166a were detected in chow diet (a mouse diet with a relatively low fat and cholesterol content) in the concentrations of $0.43,0.54$ and $0.66 \mathrm{fmol} / \mathrm{g}$ (miRNA level/weight of chow diet), respectively. However, the levels of these three miRNAs were 6-, 10- and 3-fold higher, respectively, in fresh rice than in chow diet (Figure 2A). In contrast, mammal miRNAs were expressed in chow diet but were undetectable in fresh rice (Figure 2A, insert). It is worth noting that these three plant miRNAs, MIR168a, MIR156a, and MIR166a, were detected in other foods, including Chinese cabbage (Brassica rapa pekinensis), wheat (Triticum aestivum), and potato (Solanum tuberosum) (Supplementary information, Table S3). Interestingly, plant miRNAs were stable in cooked foods (Supplementary information, Table S3). The levels of MIR168a and MIR156a were further assessed in the sera and tissues of mice fed with either a chow diet or fresh rice following $12 \mathrm{~h}$ of fasting. As shown in Figure 2B and 2C and Supplementary information, Figure S2C, the levels of plant MIR168a and MIR156a were not elevated in the serum or liver at $0.5,3$, and $6 \mathrm{~h}$ after chow diet feeding. In contrast, the levels of these two miRNAs were significantly increased in both the sera and the livers of mice fed with fresh rice for $6 \mathrm{~h}$ (Figure 2B and 2C and Supplementary information, Figure S2C). We further compared the levels of MIR168a in perfused and non-perfused organs after rice feeding and found no difference (Supplementary information, Figure S2D), which indicates that the elevation of plant miRNAs in mouse organs was not due to contamination by blood cells. In agreement with the hypothesis that exogenous plant miRNAs are derived from food intake, the levels of MIR168a were increased in the stomach and small intestine, but not in the kidney, of mice following feeding with a chow diet or fresh rice (Supplementary information, Figure S2E-S2G). Furthermore, when mice were gavage fed with total RNA extracted from fresh rice, the levels of MIR168a in mouse serum and liver were elevated $3 \mathrm{~h}$ after feeding (Figure 2D and 2E). Additionally, mice were gavage fed with synthetic MIR168a (single-stranded mature MIR168a) or synthetic methylated MIR168a because it has been reported that plant miRNAs are methylated [27]. The levels of MIR168a in mouse serum and liver were elevated $3 \mathrm{~h}$ after feeding (Figure $2 \mathrm{~F}$ and $2 \mathrm{G}$ ). To identify the form in which MI$\mathrm{R} 168 \mathrm{a}$ in a food such as rice was taken up by $\mathrm{C} 57 \mathrm{BL} / 6 \mathrm{~J}$ mice, mice were gavage fed with various forms of MIR168a, including double-stranded miRNA (dsMIR168a), precursor miRNA (pre-MIR168a), single-stranded DNA miRNA (ssDNA-MIR168a), and DNA precursor miRNA (pre-DNA-MIR168a), and the level of each form of MI$\mathrm{R} 168 \mathrm{a}$ in mouse serum was assessed after $6 \mathrm{~h}$. Interestingly, we only detected single-stranded mature MIR168a by qRT-PCR (Supplementary information, Figure S2HS2K), strongly suggesting that food-derived MIR168a is 

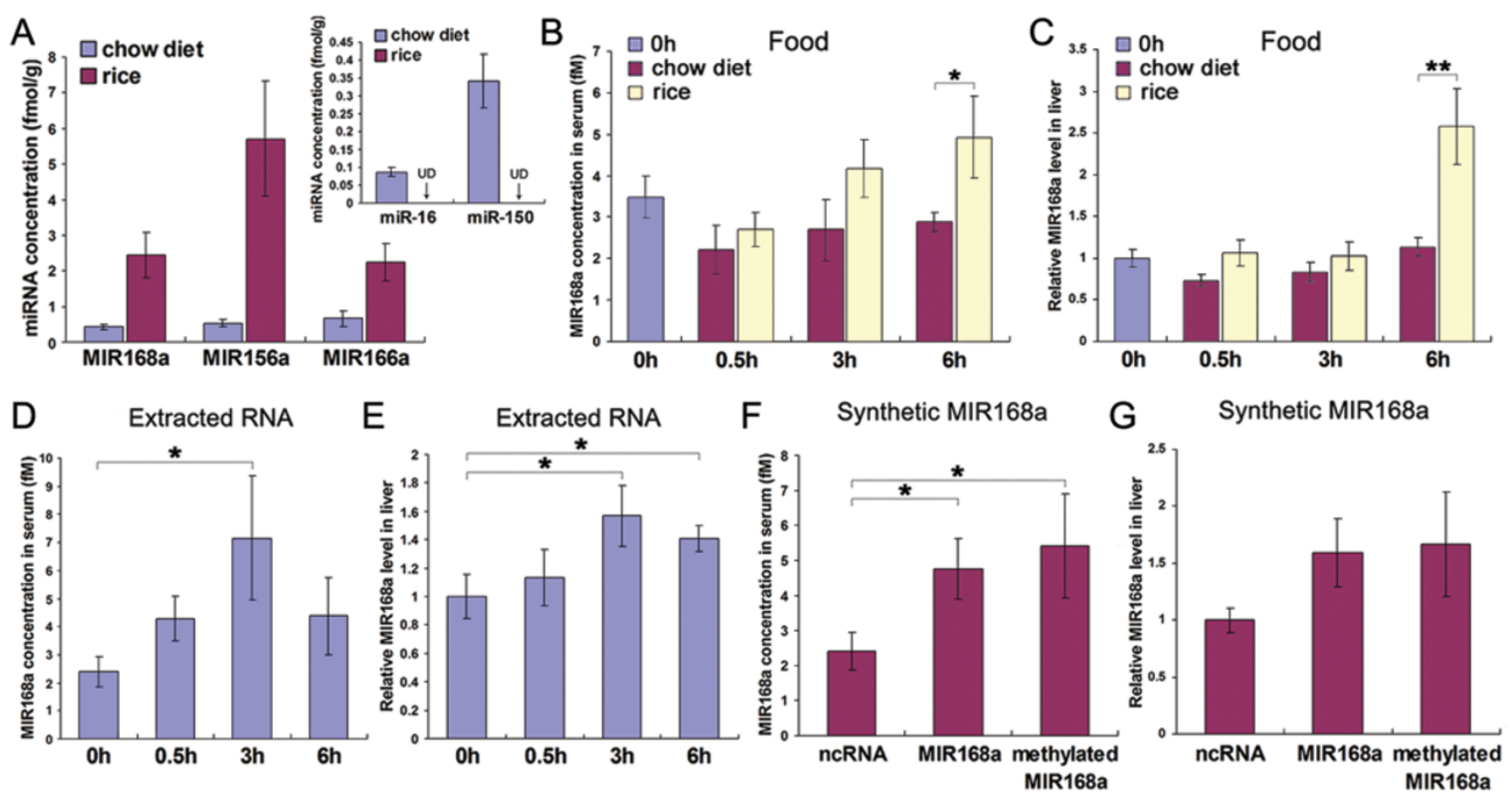

Figure 2 The exogenous mature plant miRNAs in food can pass through gastrointestinal (GI) tract and enter the sera and organs. (A) The levels of MIR168a, MIR156a, and MIR166a detected by qRT-PCR in fresh rice and chow diet ( $n=6)$. Two endogenous animal miRNAs, miR-16, and miR-150, served as controls (insert). UD, undetectable. (B, C) The levels of MIR168a in mouse serum (B) and liver (C) after feeding with fresh rice or chow diet for $0.5 \mathrm{~h}, 3 \mathrm{~h}$, or $6 \mathrm{~h}(n=8)$. The control group (named $0 \mathrm{~h}$ ) was euthanized after a 12-h of fasting. (D, E) The levels of MIR168a in mouse serum (D) and liver (E) following gavage feeding with RNA extracted from fresh rice $(n=8)$. After $0.5 \mathrm{~h}, 3 \mathrm{~h}$, or $6 \mathrm{~h}$, MIR168a levels were detected by qRT-PCR. The control group $(0 \mathrm{~h})$ was euthanized after a 12-h of fasting. (F, G) The levels of MIR168a in mouse serum (F) and liver (G) following gavage feeding with synthetic MIR168a and synthetic methylated MIR168a $(n=8)$. The control group was gavage fed with ncRNA. * $P<0.05 ;{ }^{* *} P<0.01$.

taken up in its mature form. To mimic GI tract environment, the effect of acidification on the stability of plant miRNAs and mammalian miRNAs was examined. Total RNA isolated from rice or mouse liver was adjusted to pH 2.0 and kept at $37{ }^{\circ} \mathrm{C}$ for several hours. As shown in Supplementary information, Figure S2L, acidification did not significantly affect the yield and quality of miRNAs. The majority of plant miRNAs and mammalian miRNAs can survive under acidic condition for at least $6 \mathrm{~h}$. Interestingly, the degradation rate of mammalian miRNAs under acidic condition was similar to that of their synthetic form (without 2'-O-methylated 3' ends), whereas plant miRNAs had a much slower degradation rate compared with their synthetic form (without 2'-O-methylated 3' ends), suggesting that methylation had a protective effect on the stability of plant miRNAs.

Plant MIR168a binds to exon 4 of mammalian LDLRAP1 and decreases LDLRAP1 protein level in vitro

In a previous study, it was demonstrated that a 2.5- 4.0-fold induction of secreted endogenous monocytic miR-150 in the plasma of patients with atherosclerosis could sufficiently decrease the protein level of its target gene, c-Myb, and increase cell migration in recipient endothelial cells [15]. Given that structurally functional exogenous plant miRNAs can enter human and animal sera and tissues through food intake and can be maintained at elevated levels, we hypothesized that they may play a role in regulating the functions of mammalian cells and organs. Because most plant miRNAs can act like RNA interference (RNAi), which requires a high degree of complementarity between miRNAs and target RNAs [28], we performed bioinformatic analysis to identify any sequences in the human, mouse, or rat genome with perfect or near-perfect match to MIR168a. Approximately 50 putative target genes were identified as the target genes of MIR168a (Supplementary information, Table S4). The most highly conserved sequence of a putative binding site among various species is located in exon 4 of the low-density lipoprotein receptor adapter protein 1 
(LDLRAP1) (Figure 3A). We calculated the minimum free energy of binding to be $-29.2 \mathrm{kcal} / \mathrm{mol}$. LDLRAP1 is a liver-enriched gene that plays a critical role in facilitating the removal of LDL from the circulatory system [29]. As shown in Figure 3B, transfection of HepG2 cells (a hepatocyte carcinoma cell line) with MIR168a precursor (pre-MIR168a) resulted in a 1000 -fold elevation in MIR168a, suggesting that plant pre-MIR168a can be properly processed in mammalian HepG2 cells. Additionally, the LDLRAP1 protein level in these cells was significantly reduced (Figure 3C and 3D), whereas the LDLRAP1 mRNA level was not affected (Figure $3 \mathrm{E}$ and $3 \mathrm{~F}$ ). A luciferase reporter assay was employed to demonstrate the direct binding of MIR168a to exon 4 of LDLRAP1. The same strategy has been used by others in studying miRNA targeting in coding regions [3032]. In this experiment, the wild-type (WT) or mutant (MUT) MIR168a complementary site (CS), WT or MUT LDLRAP1 binding site (BS), the human LDLRAP1 exon 4, and human LDLRAP1 coding sequence (CDS) were cloned into a luciferase reporter plasmid (Figure $3 \mathrm{G})$ and transfected into HepG2 cells combined with pre-MIR168a. As expected, luciferase reporter activity for WT was significantly reduced following transfection with pre-MIR168a, whereas the MUT luciferase reporter was unaffected by transfection with pre-MIR168a (Figure $3 \mathrm{H})$. Because the predicted MIR168a binding site is not in the $3^{\prime}$ UTR but in the open reading frame (ORF) of LDLRAP1, we further compared the efficiency of our luciferase reporter assay with those of typical reporters in which the endogenous miRNAs bind to the $3^{\prime}$ UTR of target genes. We found that the rate of the inhibition of luciferase activity by MIR168a was dependent on the ratio of pre-MIR168a to BS-containing luciferase reporter (Supplementary information, Figure S3A) and that the efficiency of MIR168a inhibition of LDLRAP1 expression was similar to the inhibition of target genes by endogenous miRNAs (miR-16, miR-21, and miR150; Supplementary information, Figure S3B). To determine whether MIR168a could directly target LDLRAP1 within its CDS, we followed the strategy proposed by others [30-32] and created a construct that expressed the full-length ORF of LDLRAP1 (Figure 3I). Furthermore, we used site-directed mutagenesis to create a variant of the LDLRAP1 construct in which the MIR168a target site was mutated while leaving the LDLRAP1 aminoacid sequence intact (Figure 3I). These constructs were co-transfected with pre-MIR168a or pre-ncRNA into 293 T cells. WT LDLRAP1 was strongly downregulated by MIR168a, whereas MIR168a had no effect on the expression of LDLRAP1 when the MIR168a target site was mutated (Figure 3J and 3K). To further explore this phenomenon, the LDLRAP1 ORF was GFP tagged at its carboxyl terminus (Figure 3L). Compared to cells transfected with ncRNA, the number of cells expressing GFP-tagged LDLRAP1 was drastically decreased in cells transfected with pre-MIR168a (Figure 3M and 3N).

Although overexpression of pre-miRNA is the conventional method to assess the function of a miRNA, we determined the direct effect of a single-stranded mature plant MIR168a on LDLRAP1 expression in HepG2 cells for two reasons. First, neither plant pri-miRNA nor plant pre-miRNA has been detected in the sera and tissues of human and animals. Second, and more importantly, single-stranded mature MIR168a is likely to be the form taken up by animals via the GI tract. By transfecting HepG2 cells with equivalent amounts of single-stranded mature plant MIR168a, we increased the level of mature MIR168a in HepG2 cells by 10000 -fold (Supplementary information, Figure 3C) and observed a concomitant reduction in LDLRAP1 (Supplementary information, Figure S3D and S3E). Similar to the results from transfection with pre-MIR168a, the overexpression of singlestranded mature MIR168a had no effect on LDLRAP1 mRNA levels (Supplementary information, Figure S3F and $\mathrm{S} 3 \mathrm{G})$. A luciferase reporter assay also demonstrated that high levels of mature MIR168a decreased luciferase activity (Supplementary information, Figure $\mathrm{S} 3 \mathrm{H}$ ), in agreement with the observation that mature MIR168a could affect LDLRAP1 expression despite a relatively low affinity for the putative binding site of LDLRAP1.

AGO2-associated mature MIR168a in Caco-2 cell-derived $M V S$ sufficiently reduces mammalian LDLRAP1 protein level in recipient HepG2 cells

In this study, we hypothesized that epithelial cells, particularly in the small intestine, might take up plant miRNAs in food, then package them into MVs and release them into the circulatory system. The secreted MVs from small intestinal epithelial cells could then deliver exogenous plant miRNA to target cells of other organs and regulate recipient cell function. To test this hypothesis, human intestinal epithelial Caco-2 cells were transfected with single-stranded mature MIR168a. As indicated by Figure 4A, the MVs released by the Caco- 2 cells were collected and used to treat HepG2 cells. The LDLRAP1 protein level in HepG2 cells was then assessed. As shown in Figure 4B, the levels of mature MIR168a were elevated by 200 -fold in MVs released by Caco- 2 cells transfected with MIR168a and by 100-fold in Caco-2 MV-treated HepG2 cells. Plant MIR168a delivered into HepG2 cells via Caco-2 MVs significantly decreased the LDLRAP1 protein level in the recipient HepG2 cells (Figure 4C and 4D), while it had no effect on LD- 
A

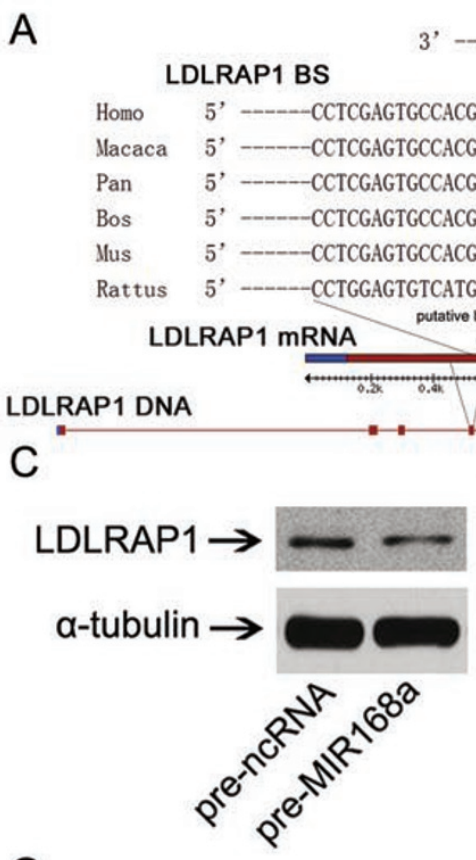

G

WT MIR168a CS (21 bp) MUT MIR168a CS (21bp) WT LDLRAPI BS (33bp) MUT LDLRAPI BS (33bp) LDLRAP1 Exon 4 (115bp) LDLRAP1 CDS (927bp)

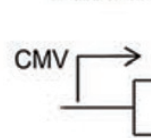
Luciferase ORF

\section{D}

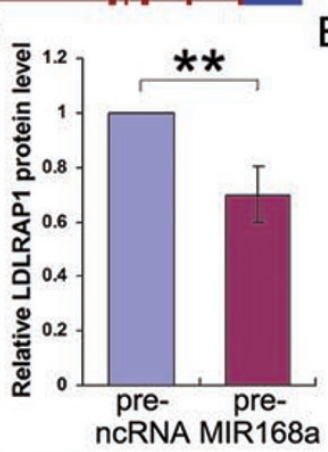

MIR168a

$$
|:|||||||||||||||||: \quad \longleftarrow \text { MFE }=-29.2 \mathrm{kcal} / \mathrm{mol}
$$

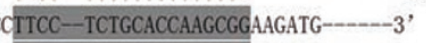

TCC-TCTGCACCAAGCGGAGATG------3,

ITC-TCTGCACCAAGCGGAAGTG----3,

- coding region

- untranslated region$$
\text { ding site }
$$

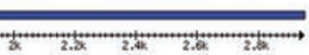

E

$\mathrm{H}$

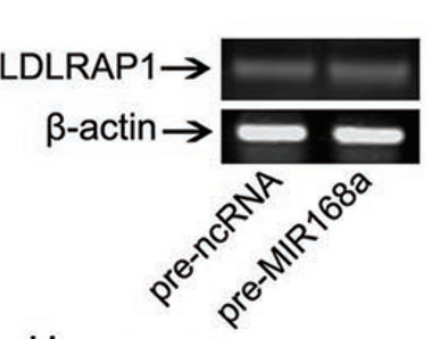

- pre-ncRNA

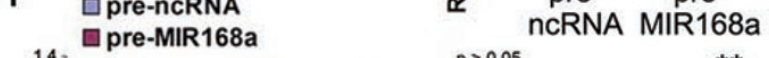

$F$

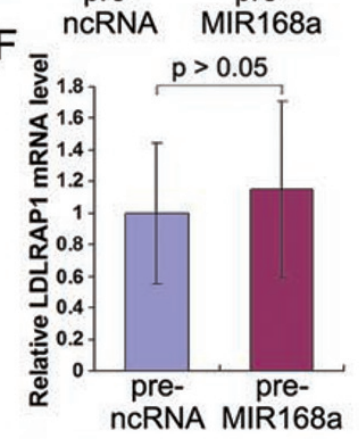

5' - GTCCCGATCTGCACCAMGCGA-3,

5' -GTCCCGATCTGCAGGTTCGCA-3,

5, -TGCCACGCCTTCCTCTGCACCAAGCGGAAGATG-3,

5' - TGCCACGCCTTCCTCTGCAGGTTCGCGAAGATG-3'

5' -GATCTC.......CTTCCTCTGCACCAAGCGG.....AAGATG-3' ' -ATGGAC.......CTTCCTCTGCACCAGGCGG…...TTCTGA-3'
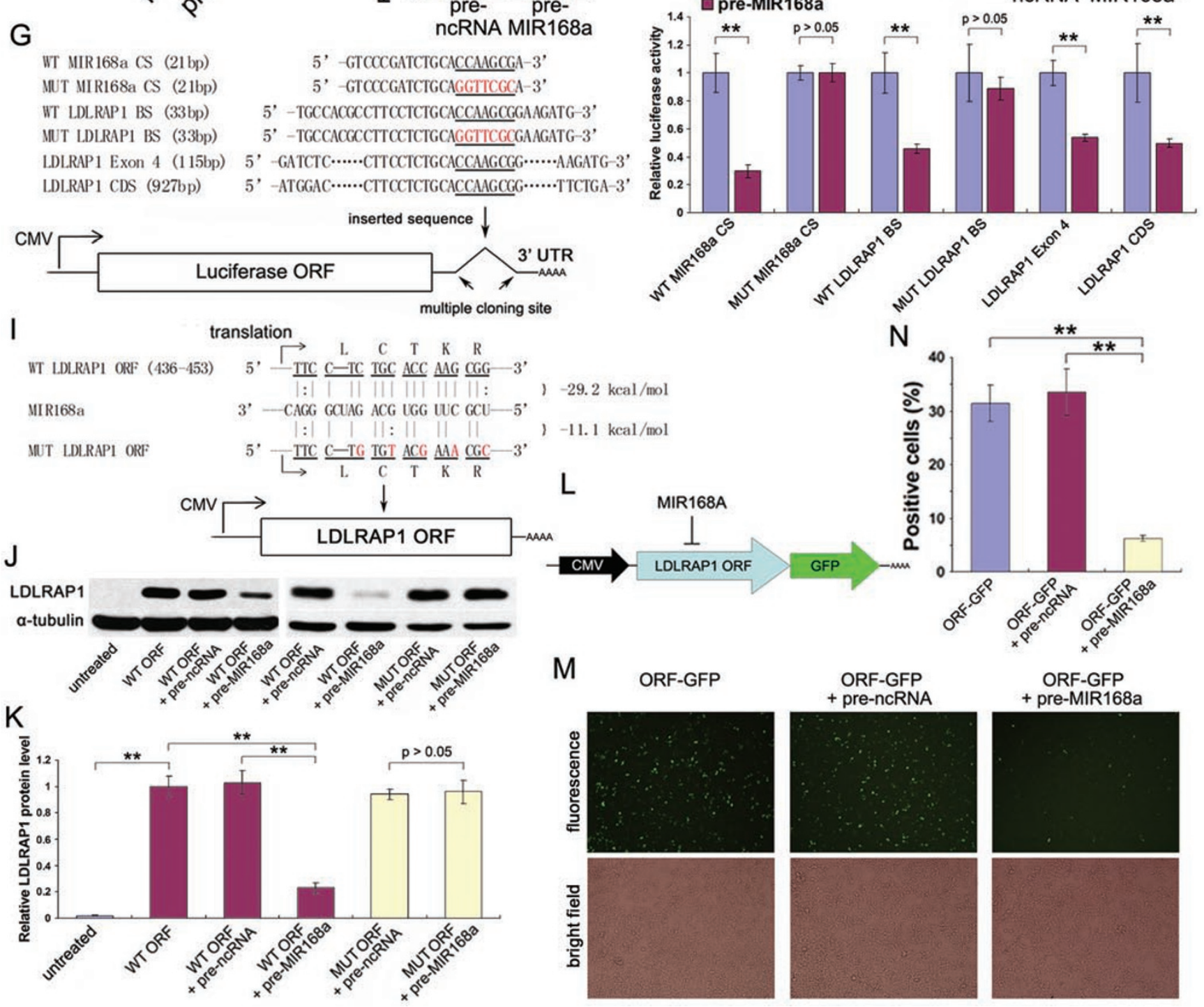

Cell Research | Vol 22 No 1 | January 2012 
Figure 3 Plant MIR168a binds to exon 4 of mammalian LDLRAP1 and decreases LDLRAP1 protein level in vitro. (A) Schematic description of the hypothesized duplexes formed by interactions between the exon 4 of LDLRAP1 and MIR168a. Paired bases are indicated by a black oval and G:U pairs are indicated by two dots. The predicted free energy of the hybrid is indicated. Note that the potential binding site of MIR168a to LDLRAP1 mRNA is highly conserved across species. (B) qRT-PCR analysis of MIR168a levels in pre-MIR168a-transfected HepG2 cells $(n=9)$. (C) Western blot analysis of LDLRAP1 protein levels in pre-MIR168a-transfected HepG2 cells. (D) The quantification of the LDLRAP1 protein expression in C $(n=9)$. (E, F) Semi-quantitative RT-PCR (E) and qRT-PCR (F) analysis of LDLRAP1 mRNA levels in pre-MIR168a-transfected HepG2 cells $(n$ $=5$ ). (G) Diagram of the luciferase reporter plasmid carrying the firefly luciferase-coding sequence attached with the wild-type (WT) or mutant (MUT) MIR168a complementary site (CS), WT, or MUT LDLRAP1 BS, LDLRAP1 exon 4, and the LDLRAP1 CDS. (H) Luciferase activities in HepG2 cells co-transfected with luciferase reporters described in G and pre-MIR168a or prencRNA $(n=9)$. (I) Constructs for the expression of the WT or MUT ORFs of LDLRAP1. (J) LDLRAP1 level in 293T cells cotransfected with WT or MUT LDLRAP1 ORFs and pre-MIR168a or pre-ncRNA. (K) The quantification of the LDLRAP1 protein expression in $\mathbf{J}(n=3)$. (L) The LDLRAP1 ORF tagged with GFP at its carboxyl terminus. (M) 293T cells were co-transfected with the GFP-tagged LDLRAP1 ORF (top) and pre-MIR168a or pre-ncRNA. GFP-positive cells were analyzed by fluorescence microscopy at $24 \mathrm{~h}$ of post-transfection. (N) The percentage of GFP-positive cells in $\mathbf{M}(n=6) .{ }^{*} P<0.05 ;{ }^{* *} P<0.01$.

LRAP1 mRNA levels (Figure 4E and 4F). Interestingly, repression of LDLRAP1 protein levels in HepG2 cells by Caco-2 MVs was dose dependent, as Caco-2 MVs containing more MIR168a decreased the expression of LDLRAP1 more effectively (Figure 4G-4I). A luciferase assay further demonstrated that plant MIR168a secreted by Caco- 2 cells could effectively enter HepG2 cells and recognize exon 4 of the LDLRAP1 transcripts. WT luciferase reporter activity was significantly reduced following incubation with MVs derived from Caco-2 cells transfected with mature MIR168a, whereas the MUT luciferase reporter was unaffected (Figure 4J). In 293T cells transfected with WT LDLRAP1 ORF, treatment with MIR168a-containing Caco-2 MVs but not ncRNAcontaining Caco-2 MVs significantly reduced the level of LDLRAP1 expression (Figure 4K and 4L). In contrast, MIR168a-containing Caco-2 MVs had no effect on the expression of the MUT LDLRAP1 ORF (Figure 4K and $4 \mathrm{~L}$ ). These results clearly indicate a possible mechanism for the action of exogenous plant miRNAs in an animal model. Moreover, because Argonaute2 (AGO2) is present in MVs and facilitates miRNA binding to its target gene via the RNA-induced silencing complex (RISC) [33], we performed an immunoprecipitation experiment to assess whether plant MIR168a was associated with mammalian AGO2 and LDLRAP1. As can be seen in Figure 4M-4O, both MIR168a (Figure 4N) and LDLRAP1 mRNA (Figure 4O) were detected in the product precipitated by an anti-AGO2 antibody from the HepG2 cells treated with Caco-2 MVs, while only MIR168a (Figure 4M) but not LDLRAP1 mRNA (Figure 4O) was detected in the antiAGO2 antibody-precipitated product from the Caco-2 MVs. As a control, endogenous mammalian miR-16 was detected with the anti-AGO2 antibody-precipitated product from both Caco-2 MVs and MV-treated HepG2 cells, but its level was not affected by MIR168a transfection
(Figure 4M and 4N). The association of MIR168a with AGO2 was additionally detected in HepG2 cells directly transfected with pre-MIR168a or mature MIR168a (Supplementary information, Figure S3I and S3J). In separate experiments, we tested whether plant miRNAs can be processed and packaged into secreted MVs by cell types other than colonic epithelial Caco-2 cells. As can be seen in Supplementary information, Figure S4, similar to Caco- 2 cells, 293T cells were able to package MIR168a into MVs and then deliver MIR168a into HepG2 cells.

Anti-MIR168a ASO reverses rice feeding-induced reduction of mouse liver LDLRAP1 protein at $6 \mathrm{~h}$ feeding

In order to determine the specificity of the inhibitory effect of MIR168a on LDLRAP1 and exclude the possibility that the reduction of LDLRAP1 protein was caused by factors other than MIR168a, an anti-MIR168a antisense oligonucleotide (ASO) was injected intravenously into mice during rice feeding. The injection of antiMIR168a ASO not only significantly reduced MIR168a levels in the livers of fresh rice-fed mice (Figure 5A), but also remarkably reversed the reduction of mouse liver LDLRAP1 by fresh rice-derived MIR168a (Figure $5 \mathrm{~B}$ and $5 \mathrm{C}$ ). Taken together, these results indicate that exogenous plant miRNAs are able to enter the serum and organs of mammals via food intake, and that plant MIR168a can bind to the nucleotide sequence located in exon 4 of mammalian LDLRAP1, leading to the inhibition of LDLRAP1 expression in vivo.

Exogenous MIR168a inhibits mouse liver LDLRAP1 expression and elevates plasma LDL-cholesterol level at 3 days after food intake

LDL is the major cholesterol-carrying lipoprotein of human plasma and plays an essential role in the pathogenesis of atherosclerosis [34]. Downregulation of 
A

Caco2 cells $\stackrel{\substack{\text { MIR168a, then harvest MVs } \\ \text { (containing MIR168a) }}}{\text { Caco2 MVs } \stackrel{\text { treat HepG2 cells }}{\longrightarrow}} \rightarrow \begin{gathered}\text { measure LDLRAP1 protein } \\ \text { and mRNA level }\end{gathered}$

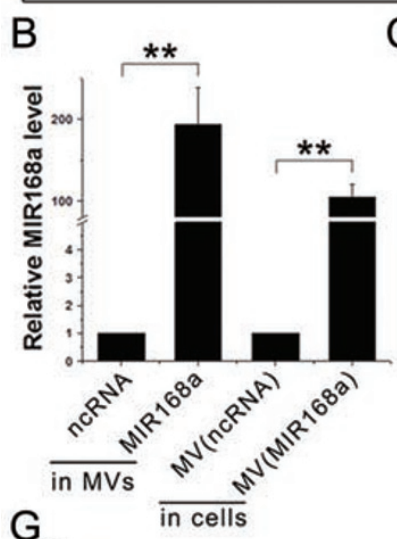

G

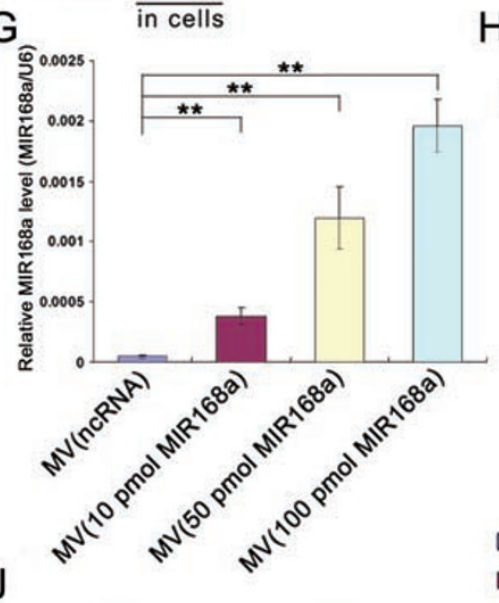

$\mathrm{H}$

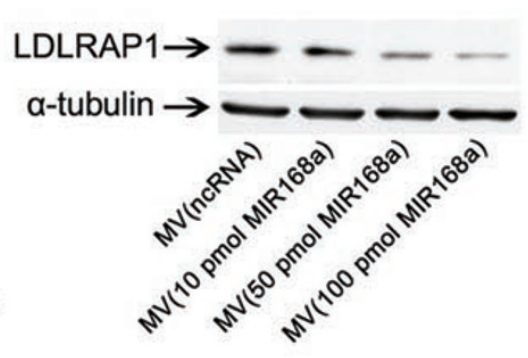

$\square M V(n c R N A)$

aMV(MIR168a) K
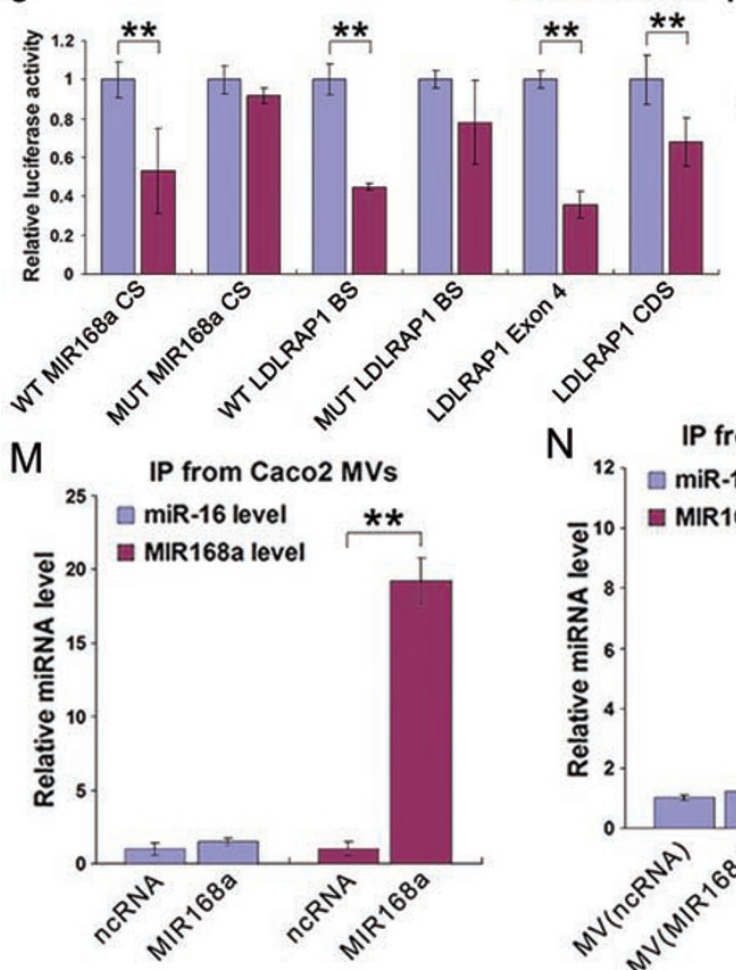

E
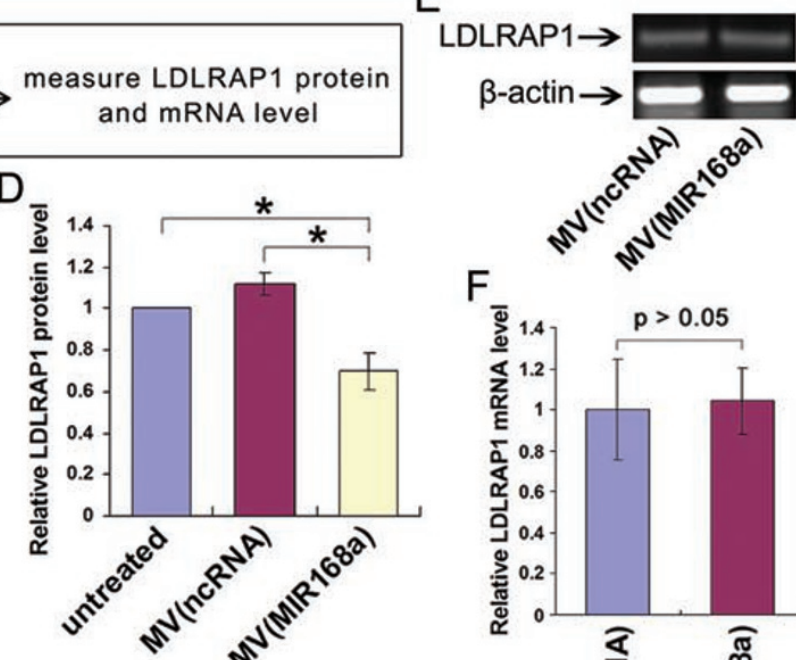

F
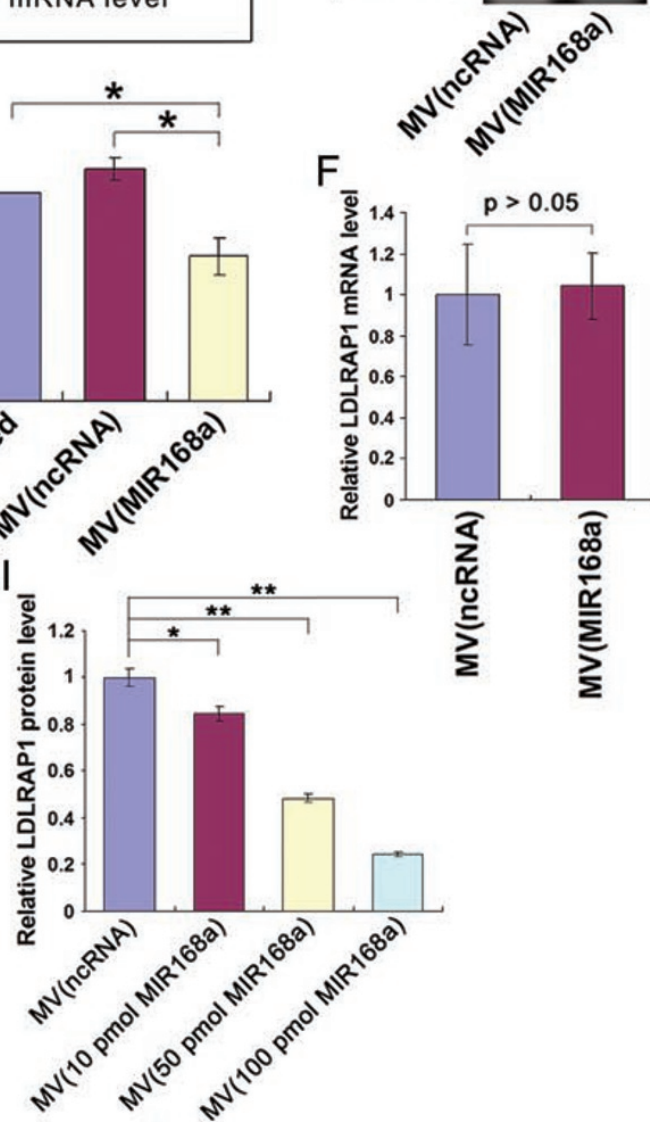

.
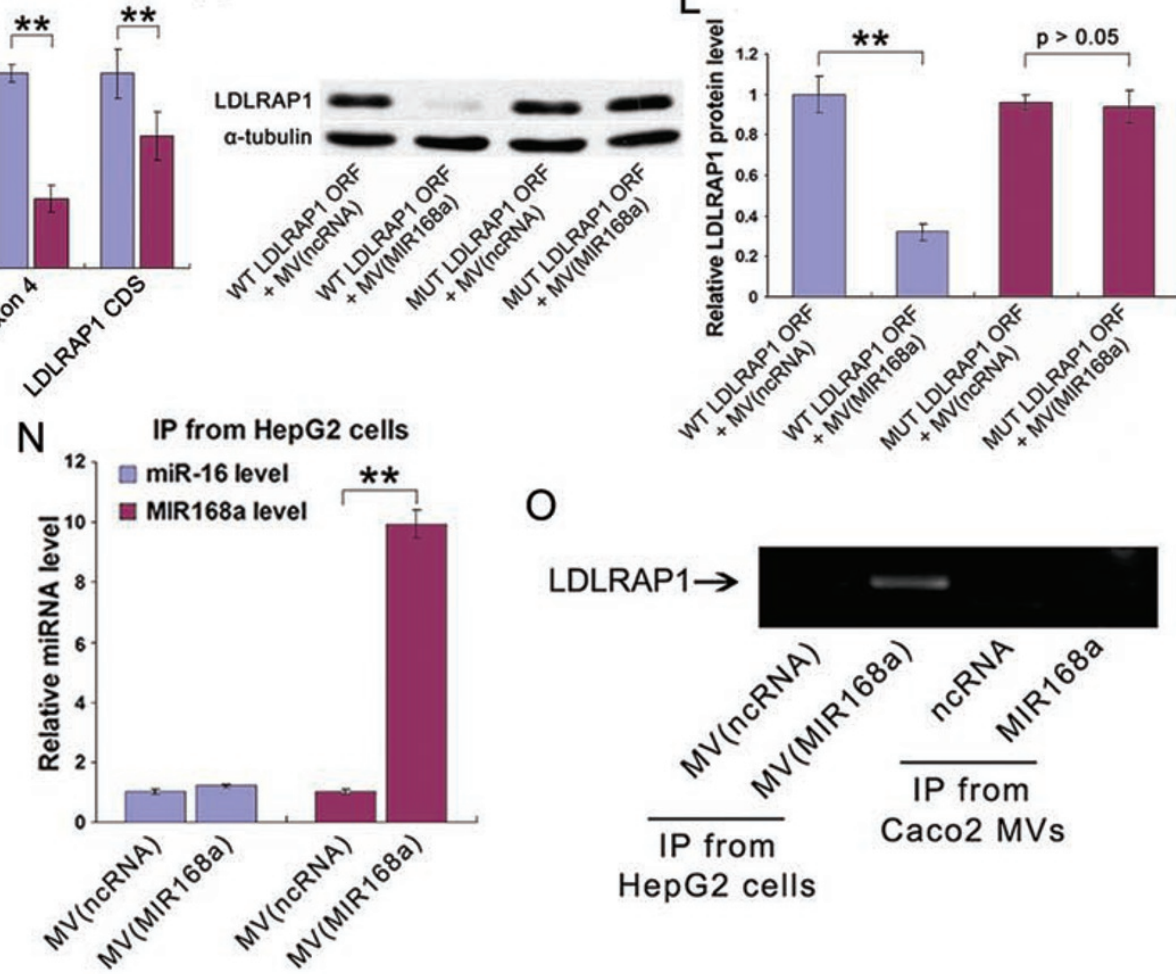

O

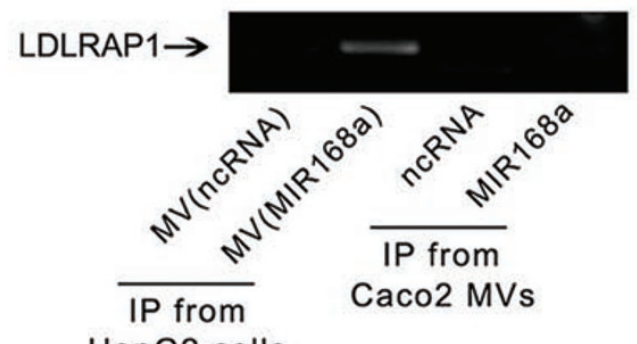

HepG2 cells 
Figure 4 AGO2-associated mature MIR168a in Caco-2 cell-derived MVs sufficiently reduces mammalian LDLRAP1 protein level in recipient HepG2 cells. (A) A flow chart of the experimental design. (B) The elevation of MIR168a in Caco-2 MVs after transfection with mature MIR168a (left) and in HepG2 cells after treatment with Caco-2 MVs (right; $n=9$ ). Caco-2 MVs were harvested after transfecting the cells with mature MIR168a or ncRNA. (C) Western blot analysis of LDLRAP1 protein levels in HepG2 cells treated with or without Caco-2 MVs. Caco-2 MVs were harvested after transfecting the cells with mature MIR168a or ncRNA. (D) The quantification of the LDLRAP1 protein expression in C $(n=6)$. (E, F) Semi-quantitative RT-PCR (E) and qRT-PCR (F) analysis of the LDLRAP1 mRNA levels in HepG2 cells treated with or without Caco-2 MVs $(n=5)$. (G, H) The levels of MIR168a (G) and LDLRAP1 protein $(\mathbf{H})$ in HepG2 cells treated with Caco-2 MVs derived from Caco-2 cells transfected with different doses $\left(10,50\right.$, or 100 pmol/10 $10^{5}$ cells) of mature MIR168a or ncRNA. (I) The quantification of the LDLRAP1 level in $\mathbf{H}(n=3)$. (J) The luciferase activities in luciferase reporter-transfected HepG2 cells treated with or without Caco-2 MVs $(n=9)$. (K) LDLRAP1 protein level in 293T cells transfected with WT or MUT LDLRAP1 ORF and then treated with Caco-2 MVs. (L) The quantification of the LDLRAP1 protein expression in $\mathbf{K}(n=3)$. (M, N) The association of MIR168a with AGO2 in Caco-2 MVs (M) and HepG2 cells treated with Caco-2 MVs (N). The levels of MIR168a and miR-16 (control) in anti-AGO2 immunoprecipitated products detected by qRT-PCR $(n=9)$. (0) The association of LDLRAP1 mRNA with AGO2 in Caco-2 MV-treated HepG2 cells or Caco-2 MVs. The LDLRAP1 mRNA in anti-AGO2 immunoprecipitated products from HepG2 cells (lanes 1 and 2) and Caco-2 MVs (lanes 3 and 4) was detected by semi-quantitative RT-PCR with 25-30 cycles. ${ }^{*} P<0.05 ;{ }^{* *} P<0.01$.

LDLRAP1 in the liver causes decreased endocytosis of LDL by liver cells and impairs the removal of LDL from plasma $[35,36]$. To assess the physiological function of food-enriched MIR168a in mammals, mice were fed with fresh rice and chow diets for 7 days after a 12-h of fasting. Body weight was not different between the two groups of mice during the experimental period (Figure $6 \mathrm{~A}$ ), although the fresh rice-fed group had an increased food intake (Figure 6B). In addition, the serum MIR168a levels of the chow diet-fed group were not significantly altered during the experimental period (Figure 6C). In contrast, there was a significant induction of serum MIR168a levels in mice after 1 day of feeding with fresh rice, and these levels remained elevated over those of the chow diet group (Figure 6C). Likewise, levels of MIR168a in the liver were increased in fresh rice-fed mice compared to chow diet-fed mice after 1 day (Figure 6D). Supplementary information, Figure S5A to S5C shows the level of MIR168a in other mouse organs after a long period of feeding. Although miR168a was initially increased in the stomach of mice fed with rice, no significant difference in MIR168a levels in the stomach, intestine, or kidney were found between chow diet- and rice-fed mice. Concomitant with a significant elevation in MIR168a levels in the livers of mice after 1 day of fresh rice feeding (Figure 6D), LDLRAP1 expression dramatically decreased in the group of fresh rice-fed mice (Figure $6 \mathrm{E}$ and $6 \mathrm{~F})$. In these experiments, LDLRAP1 levels in
A

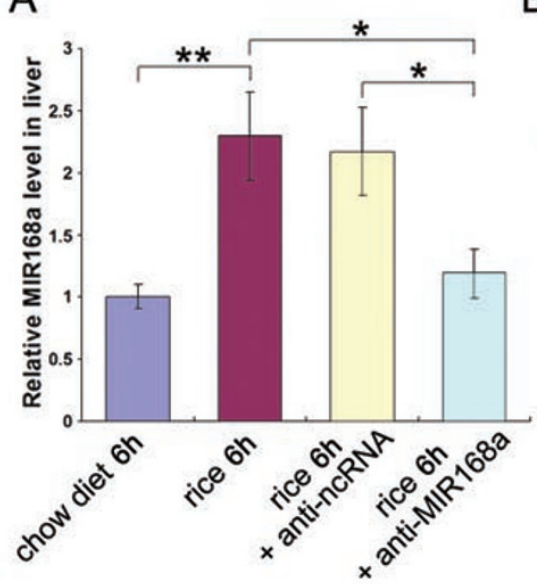

B

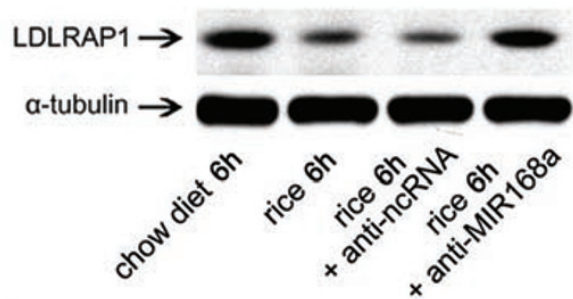

C

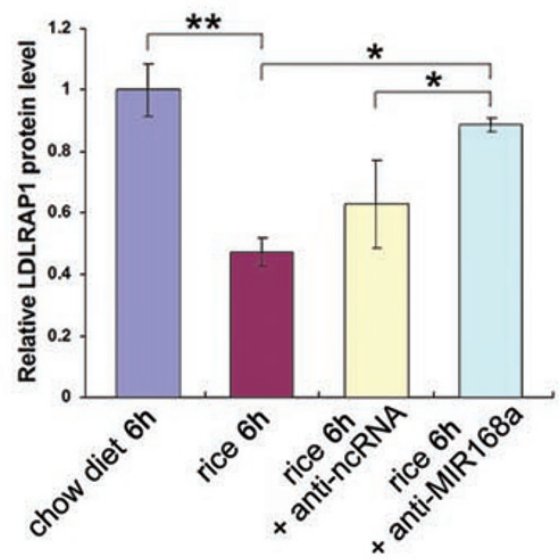

Figure 5 Anti-MIR168a ASO reverses rice feeding-induced reduction of mouse liver LDLRAP1 protein at $6 \mathrm{~h}$ feeding. (A, B) The levels of MIR168a (A) and LDLRAP1 protein (B) in mouse liver after feeding with chow diet, fresh rice, or fresh rice accompanying an intravenous injection of anti-MIR168a ASO or anti-ncRNA for $6 \mathrm{~h}(n=8)$. (C) The quantification of the LDLRAP1 level in $\mathbf{B}(n=8) .{ }^{*} P<0.05 ;{ }^{* *} P<0.01$. 

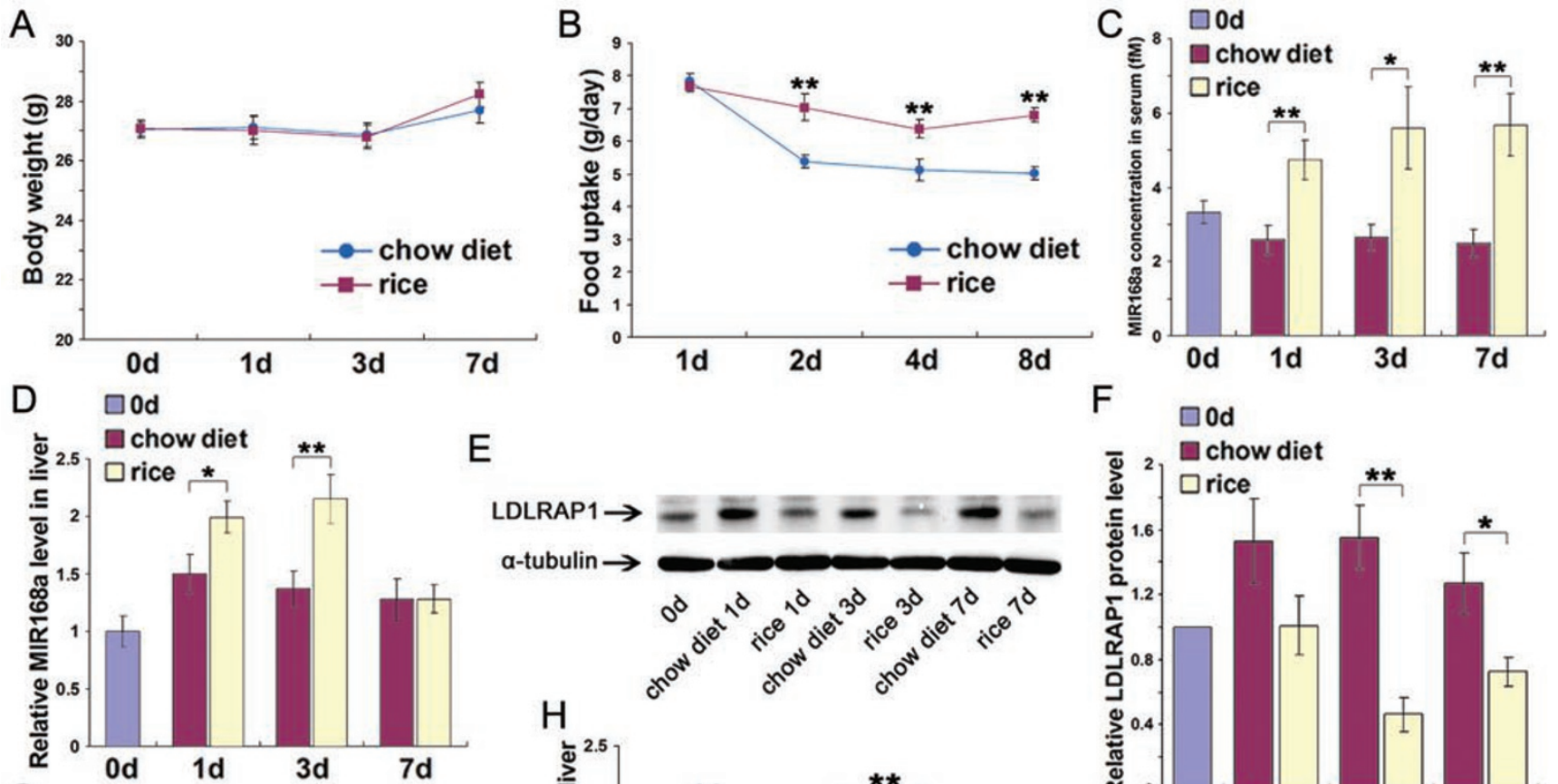

E

$\mathrm{F}$

$F \quad \square 0 d$
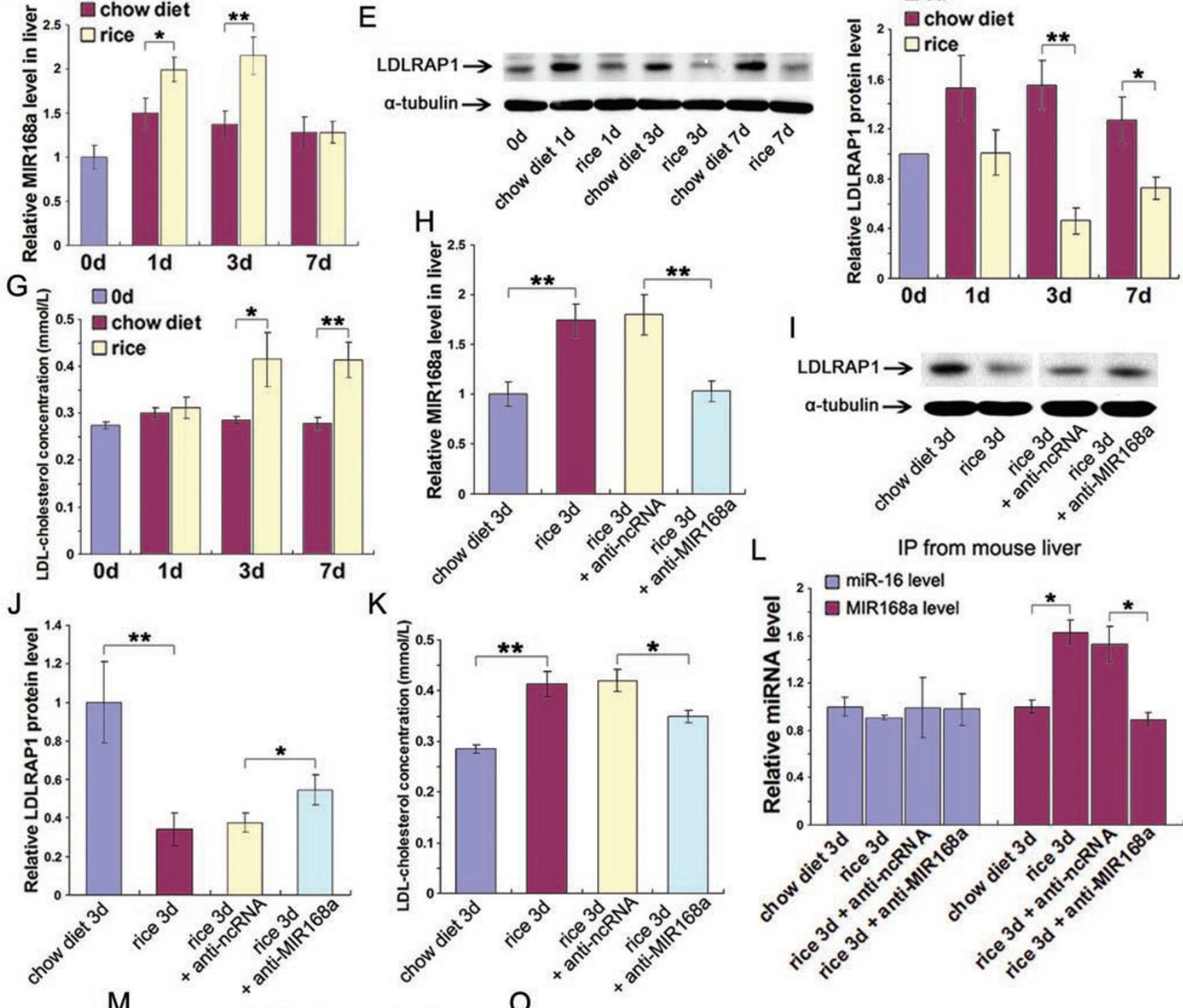

L

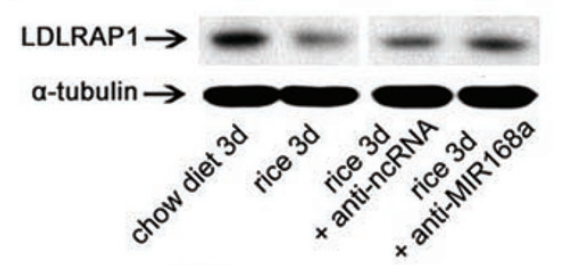

IP from mouse liver
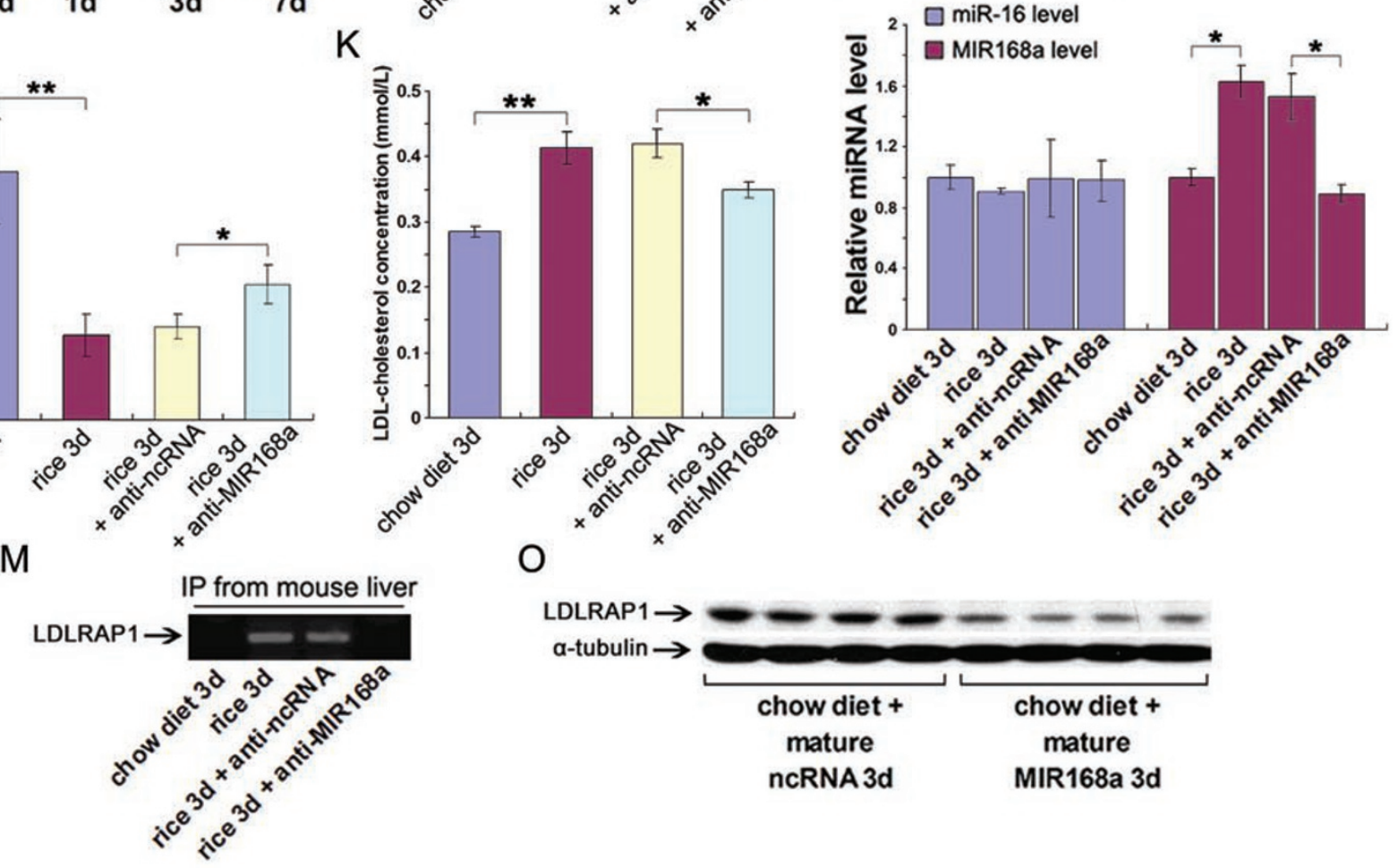

$\mathrm{O}$

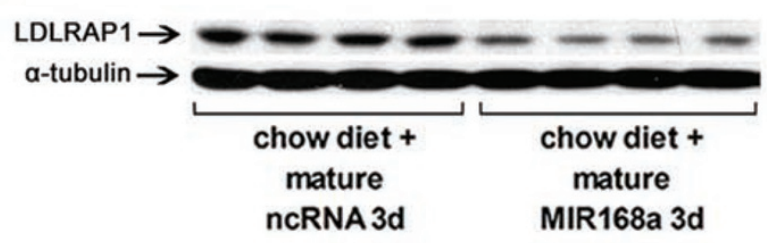



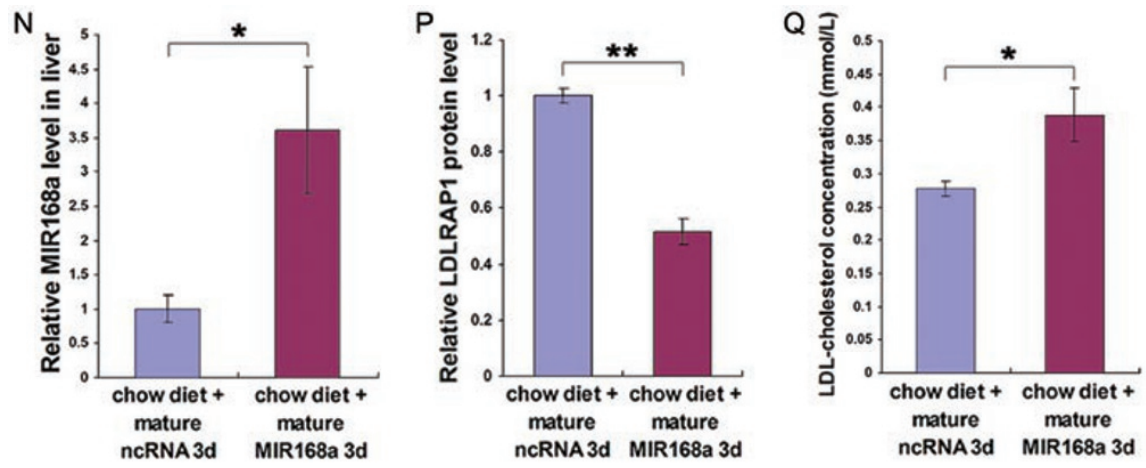

Figure 6 Exogenous MIR168a inhibits mouse liver LDLRAP1 expression and elevates plasma LDL-cholesterol level at 3 days after food intake. (A) The weight changes of mice fed with chow diet or fresh rice $(n=8)$. (B) The food intake of chow diet or fresh rice $(n=8)$. (C, D) The levels of MIR168a in mouse sera (C) and livers (D) after chow diet or fresh rice feeding $(n$ = 8). (E) The LDLRAP1 protein levels in mouse livers after chow diet or fresh rice feeding. (F) The quantification of LDLRAP1 protein expression in $\mathbf{E}(n=8)$. (G) The levels of LDL-cholesterol in mouse plasma after chow diet or fresh rice feeding $(n=8)$. $(\mathbf{H}, \mathbf{I})$ The levels of MIR168a $\mathbf{( H )}$ and LDLRAP1 protein (I) in mouse livers after 3 days of feeding with chow diet, fresh rice, or fresh rice accompanying an injection of anti-MIR168a ASO or anti-ncRNA $(n=8)$. (J) The quantification of LDLRAP1 protein expression in I $(n=8)$. (K) The levels of LDL-cholesterol in mouse plasma after 3 days of feeding with chow diet, fresh rice, or fresh rice accompanying an injection of anti-MIR168a ASO or anti-ncRNA $(n=8)$. (L) The association of MIR168a with AGO2 in mouse livers after 3 days of feeding with chow diet, fresh rice, or fresh rice plus injection of anti-MIR168a ASO or anti-ncRNA. The levels of MIR168a and miR-16 (control) in anti-AGO2 immunoprecipitated products were detected by qRT-PCR. (M) The association of LDLRAP1 mRNA with AGO2 in mouse livers after 3-day feeding. The LDLRAP1 mRNA in anti-AGO2 immunoprecipitated products was detected by semi-quantitative RT-PCR. (N, O) The levels of MIR168a (N) and LDLRAP1 protein $(\mathbf{O})$ in mouse livers after 3 days of feeding with chow diet and mature MIR168a or ncRNA $(n=8)$. (P) The quantification of LDLRAP1 protein expression in $\mathbf{O}(n=8)$. (Q) The levels of LDL-cholesterol in mouse plasma after 3 days of feeding with chow diet and mature MIR168a or ncRNA $(n=8) .{ }^{*} P<0.05 ;{ }^{* *} P<0.01$.

the control group $(0 \mathrm{~d})$ were obtained from mice that had undergone a 12 -h period of fasting. It has been found that fasting decreases LDLRAP1 expression in mouse livers, and re-feeding was sufficient to rescue this reduction (Supplementary information, Figure S5D and S5E). In agreement with the reduction of LDLRAP1 in the mouse liver, LDL levels in mouse plasma were significantly elevated on days 3 and 7 after fresh rice feeding (Figure 6G). The rice-induced elevation of mouse liver MIR168a (Figure 6H) and, more importantly, MIR168amediated reduction of liver LDLRAP1 (Figure 6I and $6 \mathrm{~J})$ was largely blocked by the administration of antiMIR168a ASO but not negative control oligonucleotides. Consistently, the rice-induced elevation of LDL levels in mouse plasma was blocked by the anti-MIR168a ASO (Figure 6K). Furthermore, the association of MIR168a with LDLRAP1 mRNA through AGO2 in mouse liver was tested. For this experiment, AGO2 protein in mouse liver following different treatment was immunoprecipitated by anti-AGO2 antibody and the levels of MIR168a (Figure 6L) and LDLRAP1 mRNA (Figure 6M) in antiAGO2 antibody IP product were assessed. As can be seen, in rice-fed mice, AGO2-associated MIR168a and LDLRAP1 mRNA in mouse livers were significantly in- creased compared to those in chow diet-fed mice and this rice feeding-induced elevation of liver AGO2-associated MIR168a and LDLRAP1 mRNA could be blocked by administration of anti-MIR168a ASO. Furthermore, by adding mature MIR168a into chow diet and feeding mice, we found that the MIR168a levels in chow diet (Supplementary information, Figure S5F), sera (Supplementary information, Figure S5G) and mouse liver (Figure $6 \mathrm{~N}$ ) were increased but the LDLRAP1 protein level in the mouse liver (Figure $6 \mathrm{O}$ and 6P) was decreased. In contrast, there is no difference of MIR156a levels between the mice after chow diet feeding with addition of MIR168a or ncRNA (Supplementary information, Figure S5G). Additionally, decreased liver LDLRAP1 levels led to an elevation in the mouse plasma LDL level (Figure 6Q). In separate experiments, we tested the link between the downregulation of mouse liver LDLRAP1 and the elevation of mouse plasma LDL level by directly decreasing liver LDLRAP1 by administration of LDLRAP1 siRNA. Downregulation of liver LDLRAP1 via LDLRAP1 siRNA (Supplementary information, Figure S6A and S6B) resulted in the elevation of plasma LDL level (Supplementary information, Figure S6C), suggesting that LDLRAP1 is responsible for plasma LDL 
clearance. However, the level of liver LDLRAP1 was not related to the levels of plasma cholesterol or triglycerides (Supplementary information, Figure S6D and S6E). Decreased levels of plasma cholesterol (Supplementary information, Figure S7A) but unchanged plasma ApoA (Supplementary information, Figure S7B) and triglyceride levels (Supplementary information, Figure S7C) further supported the hypothesis that the elevation of fresh rice-derived MIR168a in the mouse liver specifically decreased liver LDLRAP1 expression and thus caused an elevated LDL level in mouse plasma. Interestingly, no significant differences in the levels of plasma cholesterol (Supplementary information, Figure S7D) and triglycerides (Supplementary information, Figure S7E) were observed in mice after 3 days of feeding with chow diet, fresh rice, or fresh rice accompanying an injection of anti-MIR168a or anti-ncRNA oligonucleotides. As a control, mature mammalian miR-150 was added into the chow diet to feed mice. In a similar fashion, miR-150 levels in mouse liver (Supplementary information, Figure S7F) and serum (Supplementary information, Figure S7G) were significantly increased after 3 days of feeding, leading to downregulation of liver c-Myb expression (Supplementary information, Figure S7H).

\section{Discussion}

\section{Mature single-stranded plant miRNAs are stable}

Our previous study demonstrated that mature singlestranded mammalian miRNAs are stably present in serum or plasma of human and other animals [5] and that the circulating miRNAs can serve both as a class of biomarkers of diseases $[8,12]$ and as active signal molecules [15]. In the present study, we have detected stable mature single-stranded plant miRNAs in serum and tissues of mammals using both Solexa sequencing and qRT-PCR (Figure 1A, 1B, 1H and 1I). By performing Solexa sequencing and qRT-PCR after oxidation of small RNAs with periodate, we have found that the plant miRNAs identified in serum and tissues showed high levels of resistance to periodate, demonstrating that they bear 2'-O-methylated $3^{\prime}$ ends and are therefore genuine plant miRNAs (Figure 1E, 1F, 1J and $1 \mathrm{~K}$ ). Given the fact that plant miRNAs are completely exogenous, plant miRNAs detected in serum and organs of mammals should only come from food intake. Food-derived mature plant miRNAs such as MIR168a, which can be detected even after cooking the food (Supplementary information, Table S3), are quite stable in animal serum (Figure 1A-1D), suggesting that they may be also resistant to enzymatic digest in GI. Indeed, we have shown that acidification mimicking the GI tract environment did not significantly affect the stability of plant miRNAs, and that methylation may have a protective effect on plant miRNAs (Supplementary information, Figure S2L). Interestingly, pre-MIR168a and pre-MIR156a can be detected in fresh rice but not in either cooked rice or mouse serum (Supplementary information, Figure S2B), implicating that these pre-miRNAs may not be stable and able to pass through GI tract. In support of this, the gavage feeding experiment using equal amount of single-stranded miRNA (ssRNA), double-stranded miRNA (dsRNA), precursor miRNA (preRNA), singlestranded DNA form of miRNA (ssDNA), and DNA form of precursor miRNA (preDNA) clearly showed that only mature miRNA was detected in mouse serum and tissues while other forms of RNA/DNA were not detected (probably degraded; Supplementary information, Figure $\mathrm{S} 2 \mathrm{H}-\mathrm{S} 2 \mathrm{~K})$. The exclusive presence of mature MIR168a in mouse serum and tissues maybe largely dependent on its high stability compared to other forms of RNA/DNA in mouse GI to serum uptake pathway, although it cannot rule out the possibility that there is a specific pathway for mature miRNA uptake. Our results clearly demonstrate that exogenous plant mature miRNAs in food can pass through the GI tract and be transferred into the bloodstream and tissues. To our knowledge, this is the first report indicating that nucleotides with complete functional structure are resistant to digestion in the GI tract and can be delivered to other tissues.

Plant miRNAs execute their function in mammalian cells in a fashion of mammalian miRNA

Generally, mammalian miRNAs execute its function by base pairing to the complementary sites in the 3' UTR of its target genes, thus blocking the translation or triggering the degradation of the target mRNAs [1, 2]. There are only a few reports showing that mammalian miRNA can also bind to exon of the target genes [30-32]. Unlike mammalian miRNAs, it is well known that plant miRNAs act through RNAi, which requires a complete match of their nucleotide sequence with that of their target genes [28]. Plant MIR168 has been reported to be expressed widely in various species of plant $[25$, 26]. In plants, expression of ARGONAUTE1 (AGO1), the catalytic subunit of the RISC responsible for posttranscriptional gene silencing, is controlled through a feedback loop involving the MIR168 [37-39]. Here, our data showed that MIR168a decreased the LDLRAP1 protein level but did not affect the mRNA level (Figure $3 \mathrm{C}-3 \mathrm{~F}$ ), suggesting that plant MIR168a regulates mammalian LDLRAP1 translation in a fashion of mammalian functional miRNA. Results of luciferase reporter assays using multiple plasmids including the plasmid contain- 
ing partial or whole MIR168a complementary site, LDLRAP1 BS, human LDLRAP1 exon 4, and human LDLRAP1 CDS (Figure $3 \mathrm{G}$ and $3 \mathrm{H}$ ) and the results that MIR168a was also able to target the artificially expressed LDLRAP1 protein in 293T cells (Figure 3I-3K) strongly demonstrate that plant MIR168a could bind to its binding site located in exon 4 of mammalian LDLRAP1 gene, and then inhibit LDLRAP1 protein expression.

AGO2-associated mature MIR168a in MVs is the functional form in vivo

Although a very large amount of single-stranded mature plant MIR168a can affect LDLRAP1 levels in HepG2 cells (Supplementary information, Figure S3C$\mathrm{S} 3 \mathrm{H}$ ), it is unlikely that such high concentrations of mature plant miRNAs can be achieved in serum, plasma and organs of humans or animals via food intake. Our study here suggested that plant MIR168a derived from food intake might execute its function in mouse through a novel pathway. We previously demonstrated that mammalian cells could selectively pack miRNAs into MVs in response to different stimuli and then secrete these MVs into the circulation of animals or culture medium [15]. Cell-derived MVs could further efficiently deliver miRNAs into the recipient cells where these exogenous miRNAs regulate the expression of target genes and the biological functions of recipient cells [15]. In this study, we hypothesized that intestinal epithelial cells could take up plant miRNAs in food, then pack them into MVs and release the miRNA-containing MVs into the circulatory system. These MVs secreted from intestinal epithelial cells would then deliver exogenous plant miRNAs into other organs and regulate the function of recipient cells. Supporting this hypothesis, more than half of the plant MIR168a in the serum was found in MVs (Figure 1G). The results also showed that MVs contained much less amount of MIR168a but caused stronger effect on LDLRAP1 level than 'free' MIR168a did (Figure 4B-4D). The efficiency of MV-contained MIR168a was even higher than that of transfected pre-MIR168a (Figure 3C and 3D). These results indicated that MIR168a in MVs was more functionally active compared to 'free' MIR168a. The immunoprecipitation experiments in both MVs and MV-treated cells clearly showed that MIR168a was already associated with $\mathrm{AGO} 2$ in the MVs derived from Caco-2 cells (Figure 4M), from which we may draw two conclusions: (1) MIR168a in MVs is more functionally active because it has a higher affinity to AGO2 although the underneath mechanism remains unknown, and (2) plant miRNAs can use mammalian $\mathrm{AGO} 2$ to form AGO2-associated RISC and execute their functions, similar to mammalian miRNAs. The results of association of MIR168a with AGO2 in MVs, as well as the association of AGO2 with MIR168a and LDLRAP mRNA in HepG2 cells treated with Caco-2 cell MVs (Figure 4M-4O) strongly argue that exogenous plant miRNAs cross-kindomly regulate mammalian gene through a MV-mediated pathway. Previous study showed that mature miRNA had a low affinity to AGO2 [40], which might provide an explanation why an extreme elevation of mature MIR168a via the direct transfection of MIR168a was required to reduce LDLRAP1 expression.

Endogenous mammalian miRNAs have a dynamic range of expression, extending from $<1$ copy per cell to $>10000$ copies per cell, and the regulation of target genes is highly dependent on miRNA expression levels [41]. It has been shown that miRNAs expressed at low levels $(<100$ copies per cell) did not significantly repress target-containing transcripts [42], since target encounter occurs by mass action and modestly expressed miRNAs have a low probability to meet target-containing transcripts. Previous measurements of miRNAs in liver samples revealed that let-7a, miR-16, miR-20, miR-21, and miR-22 were present at 700, $3890,130,4$ 450, and 310 copies per cell, respectively [43], while miR-181a was expressed at 810 copies per cell in DP thymocytes [44] and miR-223 and miR-451 were 828 and 1972 copies per cell in CD34(+)/CD133(-) cells [45]. It has been widely demonstrated that miRNAs expressed at these concentrations are biologically active [46-49]. We calculated the amount of MIR168a that was detected in $1 \mathrm{mg}$ of total liver RNA. The amount of MIR168a was $3.2 \times 10^{-6}$ fmol (1 920 copies) per $100 \mathrm{pg}$ of total RNA, equivalent to 853 copies per cell. This quantification revealed that MIR168a is expressed in a similar concentration range in liver cells as compared with endogenous mammalian miRNAs. Besides the concentration of the miRNAs, the extent of suppression depends on a number of factors, such as the concentration of AGO2-associated miRNAs and the complementarity between the miRNA and its target site [41]. We have identified near-perfect complementarity between MIR168a and LDLRAP1 mRNA. More importantly, we have shown that exogenous plant MIR168a in recipient mammalian liver cells is AGO2 associated (Figure 6L and 6M), which represents an enriched fraction of active and functional miRNAs. Thus, MIR168a-targeting LDLRAP1 fulfills the requirement for a threshold miRNA concentration and the principle for miRNA-target recognition.

Food-derived miRNAs may serve as a novel essential nutrient

It has been widely reported that downregulation of LDLRAP1 increases plasma LDL level $[35,36]$. In the 
present study, direct reduction of LDLRAP1 in mouse liver by RNAi significantly elevated plasma LDL level (Supplementary information, Figure S6A-S6C), confirming that LDLRAP1 is a gene candidate responsible for plasma LDL removal. Interestingly, an elevated level of MIR168a but a decreased LDLRAP1 in mouse liver were detected after just $6 \mathrm{~h}$ of rice feeding (Figures 2C and 5B), indicating that exogenous plant MIR168a from food intake can quickly change mouse liver LDLRAP1 level. Continuous downregulation of mouse liver LDLRAP1 level by MIR168a through rice feeding (Figure $6 \mathrm{E}$ and $6 \mathrm{~F}$ ) resulted in an elevation of the plasma LDLcholesterol level after 3 days (Figure 6G), implicating a physiological relevance of food-derived plant MIR168a. Rice feeding-induced reduction of LDLRAP1 protein and elevation of plasma LDL-cholesterol level could be largely reversed by anti-MIR168a ASO (Figure 6I$6 \mathrm{~K})$, confirming that the rice feeding-mediated physiological alteration is specifically due to the targeting of mouse liver LDLRAP1 by MIR168a. This conclusion is also supported by the observation that chow diet with addition of mature MIR168a significantly enhanced the levels of mouse liver MIR168a (Figure 6N) and plasma LDL-cholesterol (Figure 6Q) but decreased mouse liver LDLRAP1 protein level (Figure $6 \mathrm{O}$ and 6P). Interestingly, food intake, possibly via intestinal epithelia of GI track, may represent a general pathway for uptake of food-derived or food-associated miRNAs. As shown in Supplementary information, Figure S7F-S7H, we added miR-150, an endogenous mammalian miRNA, into chow diet and fed mice with miR-150-enriched chow diet and normal chow diet, respectively. We found that miR-150 could also enter mouse liver and downregulate its target gene, c-Myb. Given that exogenous miRNAs in food or miRNAs that are 'added' into the food can enter the circulation and various organs of animals and play a role in regulating the physiological or pathophysiological conditions, food-derived exogenous miRNAs may be qualified as a novel nutrient component, like vitamins and minerals.

Previous studies have reported that the transfer of genetic material from one species to another may modulate the cellular functions of the recipient species [50, 51]. Such examples include human miRNAs targeting viral genes [50] and the translocation of host plant mRNAs into dodder (a parasitic plant) [51]. However, to our knowledge, it was still unknown whether plant miRNAs could enter mammals and modulate mammalian cell functions. By illustrating that plant miRNAs, such as MIR168a, can be delivered into animal serum and tissues through food intake and digestion and that exogenous MIR168a can target mammalian liver-specific LDL-
RAP1 in vitro and in vivo, the present study significantly extends our understanding of the role of miRNAs. With their robust stability and highly conserved sequences, secretory miRNAs can act not only in a cross-species, but also a cross-kingdom fashion. In this sense, miRNAs may represent a novel class of universal modulators that play an important role in mediating animal-plant interactions at the molecular level. Like vitamins, minerals and other essential nutrients derived from food sources, plant miRNAs may serve as a novel functional component of food and make a critical contribution to maintaining and shaping animal body structure and function. Extending from this concept, the intake of certain plant miRNAs generation after generation through a particular food source may leave an imprint on the genetic map of the human race. In conclusion, the discovery of plant miRNAs and their roles in the biology of mammalian cells and animal organs represents the first evidence of crosskingdom transfer of functionally active miRNAs and opens a new avenue to explore miRNA-mediated animalplant interactions.

\section{Materials and Methods}

\section{Reagents, cells and antibodies}

The human intestinal carcinoma cell line Caco-2 and the hepatoma cell line HepG2 were purchased from Institute of Biochemistry and Cell Biology, Shanghai Institutes for Biological Sciences, Chinese Academy of Sciences (Shanghai, China). HepG2 cells were maintained at $37{ }^{\circ} \mathrm{C}$ in a humidified $5 \% \mathrm{CO}_{2}$ incubator with Dulbecco's modified eagle medium (Gibco, CA, USA) containing $10 \%$ fetal bovine serum (FBS, Gibco), 100 units $/ \mathrm{ml}$ of penicillin, and $100 \mu \mathrm{g} / \mathrm{ml}$ of streptomycin. Caco-2 cells were grown in minimum essential medium (Gibco) containing 10\% FBS (Gibco), $100 \mathrm{units} / \mathrm{ml}$ of penicillin, and $100 \mu \mathrm{g} / \mathrm{ml}$ of streptomycin. AntiLDLRAP1 (LS-C20125) antibody was purchased from Lifespan Biosciences (Seattle, WA, USA), anti-Ago2 (ab57113) antibody was purchased from Abcam (Cambridge, MA, USA), and anti-cMyb (C19) and anti- $\alpha$-tubulin (B-7) antibodies were purchased from Santa Cruz Biotechnology (Santa Cruz, CA, USA). Synthetic RNA molecules, including pre-MIR168a, anti-MIR168a and scrambled negative control oligonucleotides (pre-ncRNA and antincRNA), were purchased from Ambion (Austin, TX, USA). Synthetic mature MIR168a oligonucleotides and scrambled negative control oligonucleotides (mature ncRNA) were purchased from Takara (Dalian, China).

\section{Serum preparation}

The recruitment of sera from 11 male (mean age $26.3 \pm 1.9$ ) and 10 female (mean age $24.2 \pm 1.3$ ) healthy Chinese, and 8 pooled samples (each pooled from 10 healthy Chinese subjects, mean age $50.9 \pm 7.9$ ) was conducted in the Healthy Physical Examination Center of the Jinling Hospital. The health condition checkup included detailed history, physical, radiological examinations, blood tests, and abdominal sonography. Subjects who showed no abnormalities during the medical checkup were enrolled. Written 
informed consent was obtained from all donors prior to the study, and the study was approved by the ethics committee of Nanjing University, China. Venous blood samples $(\sim 5 \mathrm{ml})$ were collected from each donor and placed in a serum separator tube. Samples were processed within $1 \mathrm{~h}$. Separation of the serum was accomplished by centrifugation at $800 \times g$ for $10 \mathrm{~min}$ at room temperature, followed by a $15 \mathrm{~min}$ high-speed centrifugation at $10000 \times \mathrm{g}$ at room temperature to completely remove the cell debris. The supernatant serum was recovered and stored at $-80{ }^{\circ} \mathrm{C}$ until analysis.

\section{Solexa sequencing}

The sequencing procedure was conducted as previously described [5, 52]. Briefly, total RNA was extracted from $100 \mathrm{ml}$ of serum using the Trizol Reagent (Invitrogen, Carlsbad, CA, USA) according to the manufacturer's instructions. After the PAGE purification of small RNA molecules (under 30 base pairs) and the ligation of a pair of Solexa adaptors to their $5^{\prime}$ and $3^{\prime}$ ends, the small RNA molecules were amplified using the adaptor primers for 17 cycles and the fragments of around $90 \mathrm{bp}$ (small RNA + adaptors) were isolated from PAGE gels. The purified DNA was directly used for the cluster generation and sequencing analysis using an Illumina Genome Analyzer according to the manufacturer's instructions. The image files generated by the sequencer were then processed to produce digital data. The subsequent procedures included summarizing data production, evaluating sequencing quality and depth, calculating length distribution of small RNAs, and filtrating contaminated reads. After masking the adaptor sequences, the clean reads were aligned against the miRBase database 16.0 based on the Smith-Waterman algorithm. Only candidate with identical sequence and length compared to reference miRNA was counted as miRNA matching. For normalization, the sequencing frequency of each plant miRNA was normalized to the total amount of mammalian miRNAs.

Transfection of cells with ncRNA, pre-MIR168a, or mature MIR168a

HepG2 or Caco-2 cells were seeded on 12-well plates or 10$\mathrm{mm}$ dishes overnight and transfected the following day using Lipofectamine 2000 (Invitrogen), according to the manufacturer's instructions. For the overexpression of MIR168a, 20 pmol per $1 \times$ $10^{5}$ cells of pre-MIR168a or mature MIR168a was used. Scrambled negative control pre-miRNA (pre-ncRNA) and mature ncRNA were used as controls for pre-MIR168a and mature MIR168a, respectively. Cells were harvested 24 or $48 \mathrm{~h}$ after transfection for semi-quantitative RT-PCR, real-time PCR analysis, and western blotting.

\section{MV isolation}

MVs were isolated from the cell culture medium by differential centrifugation according to previous publications [16, 17]. Briefly, after removing cells and other debris by centrifugation at $300 \times \mathrm{g}$, $1200 \times g$, and $10000 \times g$, the supernatant was centrifuged at 110 $000 \times g$ for $2 \mathrm{~h}$ (all steps were performed at $4{ }^{\circ} \mathrm{C}$ ). MVs were collected from the pellet and resuspended in FBS-free medium.

\section{$R N A$ isolation and qRT-PCR of mature miRNAs}

Total RNA was extracted from the serum, cells, or tissues using TRIzol Reagent or Trizol LS Reagent (Invitrogen) according to the manufacturer's instructions. Quantitative RT-PCR was performed using TaqMan miRNA probes (Applied Biosystems, Foster City, CA, USA) according to the manufacturer's instructions. Briefly, total RNA was reverse transcribed to cDNA using AMV reverse transcriptase (Takara) and a stem-loop RT primer (Applied Biosystems). Real-time PCR was performed using a TaqMan PCR kit and an Applied Biosystems 7300 Sequence Detection System (Applied Biosystems).

All reactions, including no-template controls, were performed in triplicate. After the reaction, the $\mathrm{C}_{\mathrm{T}}$ values were determined using fixed threshold settings. To calculate the absolute expression levels of the target miRNAs, a series of synthetic miRNA oligonucleotides at known concentrations was reverse transcribed and amplified. The absolute amount of each miRNA was then calculated in reference to the standard curve. In the experiments presented here, miRNA expression in cells was normalized to U6 snRNA, as is common in many other reports [53].

\section{Deep sequencing and qRT-PCR after oxidation of small RNAs with periodate}

The methylation status of small RNAs was evaluated by treatment of the small RNAs with sodium periodate followed by Solexa sequencing or qRT-PCR with the miScript PCR system. Periodate oxidation was performed as previously described with slight modifications [22, 54]. Briefly, total RNA was extracted from $200 \mathrm{ml}$ of human serum or $100 \mathrm{mg}$ mouse liver using the Trizol Reagent (Invitrogen) according to the manufacturer's instructions. Small RNA fractions (fewer than 30 base pairs) were enriched by PAGE purification. A $100 \mu \mathrm{l}$ mixture consisting of $20 \mu \mathrm{g}$ of small RNA fraction and $10 \mathrm{mM} \mathrm{NaIO}_{4}$ was incubated at $0{ }^{\circ} \mathrm{C}$ for $40 \mathrm{~min}$ in dark. The oxidized RNA was precipitated twice by ethanol, rinsed once with $80 \%$ ethanol, aired dried, dissolved in $\mathrm{ddH}_{2} \mathrm{O}$, and then subjected to Solexa sequencing or qRT-PCR assay. The qRT-PCR assay was conducted using the miScript PCR system (QIAGEN, Valencia, CA, USA) according to the manufacturer's instructions. Briefly, miRNAs are polyadenylated by poly(A) polymerase and subsequently converted into cDNA by reverse transcriptase with oligo-dT. The cDNA is then used for real-time PCR quantification of mature miRNAs.

\section{Northern blotting analysis}

Oligonucleotide probes complementary to mature miRNAs were end-labeled with $\gamma-{ }^{32} \mathrm{P}$-ATP using T4 Polynucleotide Kinase (Takara). Labeled probes were purified using a Sephadex G25 spin column (Roche). Total RNA was extracted from $100 \mathrm{ml}$ human serum or $40 \mathrm{ml}$ calf serum using TRIzol Reagent (Invitrogen) according to the manufacturer's instructions. Synthetic oligonucleotides (1 pmol) were loaded as positive control. Total RNA was fractionated by PAGE using a $15 \%$ denaturing polyacrylamide gel. The RNA was then transferred onto a nylon membrane (Hybond $\mathrm{N}+$, Amersham Biosciences) by electroblotting at $200 \mathrm{~mA}$ in $0.5 \times$ TBE (Tris-Borate-EDTA) buffer for $2 \mathrm{~h}$. The membrane was dried and cross-linked. A prehybridization step was performed by incubating the membrane with $10 \mathrm{ml}$ of ULTRAhyb-Oligo solution (Ambion) pre-heated to $65^{\circ} \mathrm{C}$. Prehybridization was performed for $1 \mathrm{~h}$ at $37{ }^{\circ} \mathrm{C}$ in a standard rotating hybridization oven. The radiolabeled probe was added directly to the ULTRAhyb-Oligo solution and the membrane was incubated overnight at $37^{\circ} \mathrm{C}$ with rotation in a hybridization oven. After hybridization, the membrane was washed $3 \times 10 \mathrm{~min}$ at room temperature in $2 \times \mathrm{SSC}$ (Saline-sodium 
citrate), $0.5 \%$ sodium dodecyl sulfate (SDS) and then $1 \times 15 \mathrm{~min}$ at $42{ }^{\circ} \mathrm{C}$ in $2 \times \mathrm{SSC}, 0.5 \% \mathrm{SDS}$. The membrane was wrapped in plastic wrap and exposed to an X-ray film at $-80{ }^{\circ} \mathrm{C}$.

\section{Western blot analysis}

Samples of tissues and cultured cells were lysed in a buffer (50 mM Tris-HCl pH 7.4, 150 mM NaCl, 1\% NP-40, 0.1\% SDS), sonicated $(6 \times 1.5 \mathrm{~s}, 30 \%$ power $)$, and centrifuged at $12000 \times \mathrm{g}$ for $10 \mathrm{~min}$ at $4{ }^{\circ} \mathrm{C}$. The supernatant fraction was removed, and the protein concentration was determined by BCA assay (Pierce, Rockford, USA). Aliquots of proteins ( 60 to $100 \mu \mathrm{g}$ ) were separated on $10 \%$ SDS-polyacrylamide gels (SDS-PAGE) and transferred to polyvinylidene difluoride (PVDF) membranes. The membranes were blocked for $1 \mathrm{~h}$ at room temperature with $5 \%$ non-fat milk in Tris-buffered saline (TBS) plus Tween 20 (TBST), followed by an overnight incubation at $4{ }^{\circ} \mathrm{C}$ with antibodies (diluted in blocking buffer) against LDLRAP1 and $\alpha$-tubulin. Following $3 \times 10 \mathrm{~min}$ washes with TBST, the blots were incubated at room temperature for $1 \mathrm{~h}$ with the appropriate secondary antibody conjugated to horseradish peroxidase (HRP) and detected with an enhanced chemiluminescence reagent (Cell Signaling Technology Inc., USA). The autoradiographic intensity of each band was scanned and quantified using BandScan software (Glyko Inc., Novato, CA, USA). Values were normalized to $\alpha$-tubulin and the ratio to the control values was calculated.

\section{Plasmid construction and luciferase assay}

A mammalian expression vector encoding the human LDLRAP1 ORF (pReceiver-M02-LDLRAP1) and its C-terminally GFP-tagged form ( $p$ Receiver-M03-LDLRAP1) were purchased from GeneCopoeia (Germantown, MD, USA). To introduce mutations into the MIR168a target site in the LDLRAP1 coding region, primers were designed for site-directed mutagenesis that resulted in the destruction of the MIR168a target site without altering the amino-acid sequence of LDLRAP1. The sites (LCTKR) were mutated as follows: prior to mutagenesis, CTC TGC ACC AAG CGG; following mutagenesis, CTg TGt ACg AAa CGc. To generate luciferase reporters, the amplified fragments were cloned into the 3' UTR region of the pMIR-report plasmid (Ambion). Efficient insertion was confirmed by sequencing. For luciferase reporter assays, $0.2 \mu \mathrm{g}$ of firefly luciferase reporter plasmid, $0.1 \mu \mathrm{g}$ of $\beta$-galactosidase expression vector (Ambion), and equal amounts (20 pmol) of pre-MIR168a, mature MIR168a or scrambled negative control RNA were transfected into cells in 24-well plates. The $\beta$-galactosidase vector was used as a transfection control. At $24 \mathrm{~h}$ post-transfection, cells were analyzed using a luciferase assay kit (Promega).

\section{Immunoprecipitation}

Immunoprecipitation assays were performed using a Chromatin Immunoprecipitation (ChIP) Assay Kit (Millipore) according to the manufacturer's instructions. Briefly, cells were washed three times with cold PBS $\left(4^{\circ} \mathrm{C}\right)$, scraped from each dish and then collected by centrifugation at $1000 \mathrm{rpm}$ for $5 \mathrm{~min}$ at $4{ }^{\circ} \mathrm{C}$. Cells were then resuspended in an appropriate volume of complete RIP lysis buffer. Mouse monoclonal anti-AGO2 antibody ( $5 \mu \mathrm{g})$ was used to immunoprecipitate RNA-binding proteins. After purification, immunoprecipitated RNA was analyzed by real-time RT-PCR for MIRNA168a using TaqMan miRNA probes (Applied Biosystems) according to the manufacturer's instructions or by semi-quantitative RT-PCR using primers specific for human LDLRAP1. Primer sequences for human LDLRAP1 were as follows: 5'-AGAGCCAGCACAACCAGA-3' (forward primer) and 5'-CTTGGACACCTGCCAAAA-3' (reverse primer). Primer sequences for mouse LDLRAP1 were as follows: 5'-AAGTATCTTGGTATGACGCTGGTG-3' (forward primer) and 5'-TCCTGGTTGGCTTTCTCCCT-3' (reverse primer).

\section{Animals}

All experimental animals were maintained on a C57BL/6J background on a 12-h light/dark cycle in a pathogen-free animal facility at Nanjing University. The Institutional Review Board of Nanjing University approved all housing and surgical procedures. At 10 weeks of age, each mouse was fed fresh rice total RNA $(80 \mu \mathrm{g})$, synthetic MIR168a (300 pmol), or synthetic methylated MIR168a (300 pmol) by gavage after fasting overnight. After a fixed time interval (i.e., $0.5 \mathrm{~h}, 3 \mathrm{~h}$, or $6 \mathrm{~h}$ ), serum and tissues were collected and total RNA was extracted. In a separate experiment, after fasting overnight, two groups of 10 -week-old male mice were placed on a diet of either mouse chow (the fundamental ingredients of the chow diet are listed in Supplementary information, Table S5) or fresh rice. Fresh food was administered every 3 days from a supply stored at $-20{ }^{\circ} \mathrm{C}$, and food consumption and body weight were measured. The mice were maintained on the diets for a fixed time interval (i.e., $0.5,3$, or $6 \mathrm{~h}$, or 1,3 , or 7 days), after which serum and tissues were collected. Furthermore, the mice fed on rice received tail vein injections of ncRNA or anti-MIR168a (10 nmol each). After a fixed time interval (i.e., 0.5, 3, or $6 \mathrm{~h}$, or 1,3 , or 7 days), sera and tissues were collected. Several mice were euthanized directly after overnight fasting, and their sera and tissues were collected as a control. In a separate experiment, synthetic MIR168a or ncRNA (100 pmol) were mixed evenly with $5 \mathrm{~g}$ of mouse chow. To avoid miRNA degradation and contamination, this food was prepared before feeding. qRT-PCR was used to determine the level of MIR168a in the chow diet with the addition of MIR168a or ncRNA. After fasting overnight, two groups of 10-week-old male mice were placed on a diet of mouse chow plus either MIR168a or ncRNA. Fresh food was administered every day, and food consumption and body weight were measured. Generally, mouse consumes 5-7 g of food per day. The mice were maintained on the diets for 3 days, after which serum and tissues were collected.

\section{Analysis of serum lipids and lipoproteins}

Serum lipids and lipoproteins were assayed using commercially available kits and a clinical chemistry analyzer (HITACHI 7600, Hitachi Koki Co. Ltd., Hitachinaka City, Japan). Reagent kits for total cholesterol and triglycerides were obtained from Randox Laboratories, Ltd. (Crumlin, UK) and reagent kits for LDL cholesterol and lipoprotein A were obtained from Daiichi Pure Chemicals (Japan).

\section{Bioinformatic analysis}

Full-length cDNAs of the human genes were obtained from the NCBI Genebank database. A program was developed and implemented to identify MIR168a-matched sites in the entire CDS/UTR of the transcripts. This program used several common criteria to determine whether a transcript was a target for MIR168a. The first 
criterion for target recognition named "seed rules" was base pairing between the "seed" (the core sequence that encompassed the first 2 to 8 bases of the mature miRNA) and the target [55]. Second, the free energy of the hybrid was expected to be within the range of the authentic miRNA-target pairs, typically lower than $-17 \mathrm{kcal} / \mathrm{mol}$. Third, an optional rule for target prediction required interspecies conservation of the putative binding sites. Using these rules, $\sim 50$ human genes were identified as putative targets of MIR168a.

\section{Statistical analysis}

All photo images of western blotting and semi-quantitative RTPCR are representative of at least three independent experiments. Real-time PCR were performed in triplicate and each experiment was repeated several times. Data shown are presented as the mean \pm SEM of at least three independent experiments; differences are considered statistically significant at $P<0.05$, using a Student's $t$-test.

\section{Acknowledgments}

We thank Dr Daniel Wu (Yale University, USA) and Dr ZhengGang Liu (Cancer Research Institute, NIH, USA) for critical reading of the manuscript and discussion of data. This work was supported by grants from the National Natural Science Foundation of China (90813035, 30890044, 30771036, 30772484, 30725008, 30890032, 31071232, 31000323, and 90608010), the National Basic Research Program of China (973 Program 2006CB503909, $2007 \mathrm{CB} 815701,2007 \mathrm{CB} 815703$, 2007CB815705, and 2007 CB815804; 863 Program 2006AA02Z177 and 2006AA10A121), and the Natural Science Foundation of Jiangsu Province (BK2008021).

\section{References}

1 Bartel DP. MicroRNAs: genomics, biogenesis, mechanism, and function. Cell 2004; 116:281-297.

2 He L, Hannon GJ. MicroRNAs: small RNAs with a big role in gene regulation. Nat Rev Genet 2004; 5:522-531.

3 Calin GA, Croce CM. MicroRNA signatures in human cancers. Nat Rev Cancer 2006; 6:857-866.

4 Esquela-Kerscher A, Slack FJ. Oncomirs - microRNAs with a role in cancer. Nat Rev Cancer 2006; 6:259-269.

5 Chen X, Ba Y, Ma L, et al. Characterization of microRNAs in serum: a novel class of biomarkers for diagnosis of cancer and other diseases. Cell Res 2008; 18:997-1006.

6 Mitchell PS, Parkin RK, Kroh EM, et al. Circulating microRNAs as stable blood-based markers for cancer detection. Proc Natl Acad Sci USA 2008; 105:10513-10518.

7 Gilad S, Meiri E, Yogev Y, et al. Serum microRNAs are promising novel biomarkers. PLoS One 2008; 3:e3148.

8 Li LM, Hu ZB, Zhou ZX, et al. Serum microRNA profiles serve as novel biomarkers for HBV infection and diagnosis of HBV-positive hepatocarcinoma. Cancer Res 2010; 70:97989807.

9 Ng EK, Chong WW, Jin H, et al. Differential expression of microRNAs in plasma of patients with colorectal cancer: a potential marker for colorectal cancer screening. Gut 2009;
58:1375-1381.

10 Resnick KE, Alder H, Hagan JP, Richardson DL, Croce CM, Cohn DE. The detection of differentially expressed microRNAs from the serum of ovarian cancer patients using a novel real-time PCR platform. Gynecol Oncol 2009; 112:55-59.

11 Wang K, Zhang S, Marzolf B, et al. Circulating microRNAs, potential biomarkers for drug-induced liver injury. Proc Natl Acad Sci USA 2009; 106:4402-4407.

12 Zhang $\mathrm{C}$, Wang $\mathrm{C}$, Chen $\mathrm{X}$, et al. Expression profile of microRNAs in serum: a fingerprint for esophageal squamous cell carcinoma. Clin Chem 2010; 56:1871-1879.

13 Cocucci E, Racchetti G, Meldolesi J. Shedding microvesicles: artefacts no more. Trends Cell Biol 2009; 19:43-51.

14 Thery C, Zitvogel L, Amigorena S. Exosomes: composition, biogenesis and function. Nat Rev Immunol 2002; 2:569-579.

15 Zhang Y, Liu D, Chen X, et al. Secreted monocytic miR-150 enhances targeted endothelial cell migration. Mol Cell 2010; 39:133-144.

16 Skog J, Wurdinger T, van Rijn S, et al. Glioblastoma microvesicles transport RNA and proteins that promote tumour growth and provide diagnostic biomarkers. Nat Cell Biol 2008; 10:1470-1476.

17 Valadi H, Ekstrom K, Bossios A, Sjostrand M, Lee JJ, Lotvall JO. Exosome-mediated transfer of mRNAs and microRNAs is a novel mechanism of genetic exchange between cells. Nat Cell Biol 2007; 9:654-659.

18 Collino F, Deregibus MC, Bruno S, et al. Microvesicles derived from adult human bone marrow and tissue specific mesenchymal stem cells shuttle selected pattern of miRNAs. PLoS One 2010; 5:e11803.

19 Meckes DG Jr, Shair KH, Marquitz AR, Kung CP, Edwards $\mathrm{RH}$, Raab-Traub N. Human tumor virus utilizes exosomes for intercellular communication. Proc Natl Acad Sci USA 2010; 107:20370-20375.

20 Ogawa R, Tanaka C, Sato M, et al. Adipocyte-derived microvesicles contain RNA that is transported into macrophages and might be secreted into blood circulation. Biochem Biophys Res Commun 2010; 398:723-729.

21 Pegtel DM, Cosmopoulos K, Thorley-Lawson DA, et al. Functional delivery of viral miRNAs via exosomes. Proc Natl Acad Sci USA 2010; 107:6328-6333.

22 Yu B, Yang Z, Li J, et al. Methylation as a crucial step in plant microRNA biogenesis. Science 2005; 307:932-935.

23 Grimson A, Srivastava M, Fahey B, et al. Early origins and evolution of microRNAs and Piwi-interacting RNAs in animals. Nature 2008; 455:1193-1197.

24 Hartig JV, Forstemann K. Loqs-PD and R2D2 define independent pathways for RISC generation in Drosophila. Nucleic Acids Res 2011; 39:3836-3851.

25 Gazzani S, Li M, Maistri S, et al. Evolution of MIR168 paralogs in Brassicaceae. BMC Evol Biol 2009; 9:62.

26 Jia X, Mendu V, Tang G. An array platform for identification of stress-responsive microRNAs in plants. Methods Mol Biol 2010; 639:253-269.

27 Chen X. MicroRNA biogenesis and function in plants. FEBS Lett 2005; 579:5923-5931.

$28 \mathrm{Du}$ T, Zamore PD. microPrimer: the biogenesis and function of microRNA. Development 2005; 132:4645-4652.

29 Garcia CK, Wilund K, Arca M, et al. Autosomal recessive 
hypercholesterolemia caused by mutations in a putative LDL receptor adaptor protein. Science 2001; 292:1394-1398.

30 Huang S, Wu S, Ding J, et al. MicroRNA-181a modulates gene expression of zinc finger family members by directly targeting their coding regions. Nucleic Acids Res 2010; 38:72117218.

31 Qin W, Shi Y, Zhao B, et al. miR-24 regulates apoptosis by targeting the open reading frame (ORF) region of FAF1 in cancer cells. PLoS One 2010; 5:e9429.

32 Tay Y, Zhang J, Thomson AM, Lim B, Rigoutsos I. MicroRNAs to Nanog, Oct4 and Sox 2 coding regions modulate embryonic stem cell differentiation. Nature 2008; 455:11241128.

33 Gibbings DJ, Ciaudo C, Erhardt M, Voinnet O. Multivesicular bodies associate with components of miRNA effector complexes and modulate miRNA activity. Nat Cell Biol 2009; 11:1143-1149.

34 Goldstein JL, Brown MS. The low-density lipoprotein pathway and its relation to atherosclerosis. Annu Rev Biochem 1977; 46:897-930.

35 Wilund KR, Yi M, Campagna F, et al. Molecular mechanisms of autosomal recessive hypercholesterolemia. Hum Mol Genet 2002; 11:3019-3030.

36 Zuliani G, Arca M, Signore A, et al. Characterization of a new form of inherited hypercholesterolemia: familial recessive hypercholesterolemia. Arterioscler Thromb Vasc Biol 1999; 19:802-809.

37 Mallory AC, Vaucheret H. ARGONAUTE 1 homeostasis invokes the coordinate action of the microRNA and siRNA pathways. EMBO Rep 2009; 10:521-526.

38 Vaucheret H, Mallory AC, Bartel DP. AGO1 homeostasis entails coexpression of MIR168 and AGO1 and preferential stabilization of miR168 by AGO1. Mol Cell 2006; 22:129-136.

39 Vaucheret H, Vazquez F, Crete P, Bartel DP. The action of ARGONAUTE1 in the miRNA pathway and its regulation by the miRNA pathway are crucial for plant development. Genes Dev 2004; 18:1187-1197.

40 Lima WF, Wu H, Nichols JG, Sun H, Murray HM, Crooke ST. Binding and cleavage specificities of human Argonaute2. J Biol Chem 2009; 284:26017-26028.

41 Brown BD, Naldini L. Exploiting and antagonizing microRNA regulation for therapeutic and experimental applications. Nat Rev Genet 2009; 10:578-585.

42 Brown BD, Gentner B, Cantore A, et al. Endogenous microRNA can be broadly exploited to regulate transgene expression according to tissue, lineage and differentiation state. Nat Biotechnol 2007; 25:1457-1467.

43 Chen C, Ridzon DA, Broomer AJ, et al. Real-time quantification of microRNAs by stem-loop RT-PCR. Nucleic Acids Res 2005; 33:e179.

44 Neilson JR, Zheng GX, Burge CB, Sharp PA. Dynamic regulation of miRNA expression in ordered stages of cellular development. Genes Dev 2007; 21:578-589.

45 Bissels U, Wild S, Tomiuk S, et al. Absolute quantification of microRNAs by using a universal reference. RNA 2009; 15:2375-2384.

46 Meng F, Henson R, Wehbe-Janek H, Ghoshal K, Jacob ST, Patel T. MicroRNA-21 regulates expression of the PTEN tumor suppressor gene in human hepatocellular cancer. Gastroenterology 2007; 133:647-658.

47 Shimizu S, Takehara T, Hikita H, et al. The let-7 family of microRNAs inhibits Bcl-xL expression and potentiates sorafenib-induced apoptosis in human hepatocellular carcinoma. J Hepatol 2010; 52:698-704.

48 Tomimaru Y, Eguchi H, Nagano H, et al. MicroRNA-21 induces resistance to the anti-tumour effect of interferonalpha/5-fluorouracil in hepatocellular carcinoma cells. $\mathrm{Br} \mathrm{J}$ Cancer 2010; 103:1617-1626.

49 Zhang J, Yang Y, Yang T, et al. microRNA-22, downregulated in hepatocellular carcinoma and correlated with prognosis, suppresses cell proliferation and tumourigenicity. Br J Cancer 2010; 103:1215-1220.

50 Jopling CL, Yi M, Lancaster AM, Lemon SM, Sarnow P. Modulation of hepatitis $\mathrm{C}$ virus RNA abundance by a liverspecific MicroRNA. Science 2005; 309:1577-1581.

51 Roney JK, Khatibi PA, Westwood JH. Cross-species translocation of mRNA from host plants into the parasitic plant dodder. Plant Physiol 2007; 143:1037-1043.

52 Chen X, Li Q, Wang J, et al. Identification and characterization of novel amphioxus microRNAs by Solexa sequencing. Genome Biol 2009; 10:R78.

53 Jiang J, Lee EJ, Gusev Y, Schmittgen TD. Real-time expression profiling of microRNA precursors in human cancer cell lines. Nucleic Acids Res 2005; 33:5394-5403.

54 Ohara T, Sakaguchi Y, Suzuki T, Ueda H, Miyauchi K. The 3' termini of mouse Piwi-interacting RNAs are 2'-O-methylated. Nat Struct Mol Biol 2007; 14:349-350.

55 Smalheiser NR, Torvik VI. Complications in mammalian microRNA target prediction. Methods Mol Biol 2006; 342:115127.

(Supplementary information is linked to the online version of the paper on the Cell Research website.) 